\title{
Pomeron fan diagrams in perturbative QCD
}

\author{
J. Bartels ${ }^{a}$ and M.A. Braun ${ }^{b}$ \\ ${ }^{a}$ II.Institut fuer Theoretische Physik, Universität Hamburg, \\ Luruper Chaussee 149, D-22761 Hamburg, Germany \\ ${ }^{b}$ Department of High Energy Physics, Saint-Petersburg State University, \\ 198504 S.Petersburg, Russia \\ E-mail: jochen.bartels@desy.de, mibraun@yandex.ru
}

\begin{abstract}
Within QCD reggeon field theory we study the formation of two subsequent triple pomeron vertices in the process $\mathrm{P} \rightarrow \mathrm{PP} \rightarrow \mathrm{PPP}$. We make use of an earlier investigation [1] of the six-reggeon amplitude in deep inelastic scattering and show that in the large- $N_{c}$ limit pomeron fan diagrams emerge with the same triple pomeron vertex in all places. We thus confirm the BK-equation, but we also find additional terms related to the reggeization of the gluon, and we discuss their potential significance. Our analysis also includes the general pomeron $\rightarrow$ two odderon vertex: a particular version of this vertex has been included into earlier generalizations the BK equation.
\end{abstract}

Keywords: Deep Inelastic Scattering (Phenomenology), QCD Phenomenology

ARXIV EPRINT: 1711.04703 


\section{Contents}

1 Introduction 1

2 The $2 \rightarrow 4$ reggeized gluon transition: the triple-pomeron vertex $V_{4} \quad 4$

2.1 Two and three gluon states coupled to the $q \bar{q}$ loop 4

2.2 The triple pomeron vertex 6

2.3 The topological derivation 9

$3 \quad$ The transition $2 \rightarrow 6 \quad 11$

4 The large- $N_{c}$ limit of $D_{6} \quad 15$

$\begin{array}{lll}4.1 & \text { New reggeizing pieces } & 15\end{array}$

$\begin{array}{ll}4.2 & \text { The large- } N_{c} \text { limit of eq. (3.9) } \\ \end{array}$

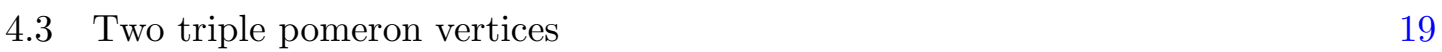

4.4 Reggeizing pieces in the four and six giuon amplitudes $D_{4}$ and $D_{6} \quad 22$

5 Discussion $\quad 26$

\section{Introduction}

Rather long time ago in the study of the interaction of a colorless projectile with two colorless targets the triple-pomeron vertex $\Gamma$ was constructed, both within QCD reggeon field theory ${ }^{1}[2,3]$ and within the dipole picture $[4,5]$. In figure 1 we illustrate the basic process in which the triple pomeron vertex appears: the scattering of a projectile (virtual photon) on two targets, described by the interaction of three BFKL ladders which are coupled to the triple pomeron vertex.

In momentum space the triple pomeron vertex is given by a somewhat lengthy function $V_{4 \leftarrow 2}^{a_{1} a_{2} a_{3} a_{4} \mid b_{1} b_{2}}\left(k_{1}, k_{2}, k_{3}, k_{4} \mid q_{1}, q_{2}\right)[1]$ which depends upon the color and the transverse momenta of the constituent reggeons of the incomjng pomeron and the two outgoing pomerons. Switching via Fourier transform to the space of transverse coordinates and taking the large$N_{c}$ limit, the expression takes the much simpler simple form [6, 7]:

$$
\begin{aligned}
\int d^{2} r_{1} d^{2} r_{2} d^{2} r_{3} P\left(r_{1}, r_{2}\right) P\left(r_{2} r_{3}\right) \Gamma\left(r_{1}, r_{2}, r_{3}\right) P\left(r_{1}, r_{3}\right) \\
=-\frac{g^{4} N_{c}}{4 \pi^{3}} \int d^{2} r_{1} d^{2} r_{2} d^{2} r_{3} P\left(r_{1}, r_{2}\right) P\left(r_{2} r_{3}\right) \frac{r_{13}^{2} \nabla_{1}^{2} \nabla_{3}^{2}}{r_{12}^{2} r_{23}^{2}} P\left(r_{1}, r_{3}\right) .
\end{aligned}
$$

\footnotetext{
${ }^{1}$ In our definition, QCD reggeon field theory is defined as the theory of interacting reggeized gluons. The BFKL pomeron appears as a bound state of (at least) two reggeized gluons.
} 


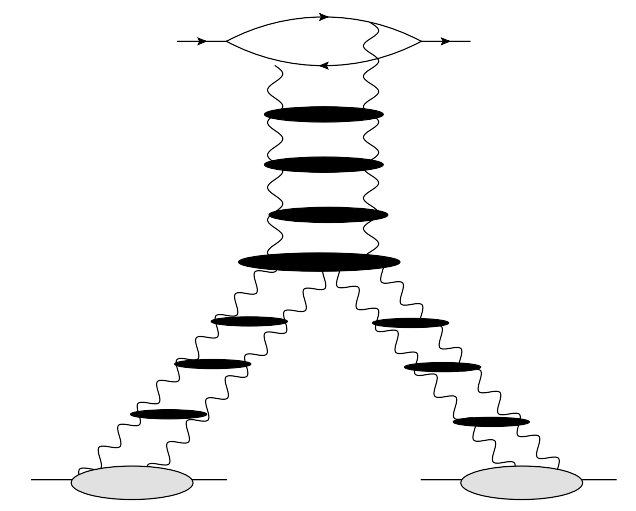

Figure 1. The triple pomeron vertex.

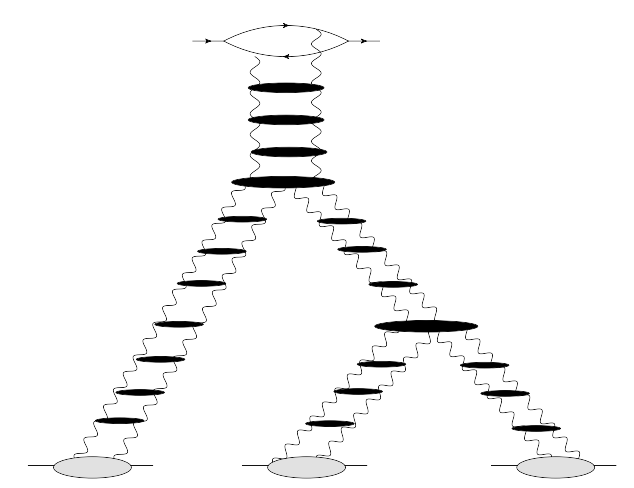

Figure 2. pomeron fan diagrams.

Here $P\left(r_{1}, r_{3}\right)$ denotes the (upper) incoming pomeron, $P\left(r_{1}, r_{2}\right)$ and $P\left(r_{2}, r_{3}\right)$ the two (lower) outgoing pomerons. They depend upon the transverse coordinates of their constituent reggeons, and $r_{i k}=r_{i}-r_{k}$. (we have suppressed the color labels and the rapidity dependence of the pomerons). This triple pomeron vertex plays an important role in applications, since it serves as a fundamental building block in constructing a theory of interacting pomerons, which is expected to describe strong interactions in the Regge kinematics. In particular, it lies at the origin of the structure functions of the heavy nuclei, as given by the Balitski-Kovchegov equation $[8,9]$ which sums pomeron fan diagrams (figure 2). In this nonlinear evolution equation this vertex represents the nonlinear part of the kernel. It is also responsible for pomeron loops, in particular the pomeron the self-mass (figure 3), which was calculated in [10-13]. In this calculation also the inverted vertex $\mathrm{PP} \rightarrow \mathrm{P}$ was introduced.

In the framework of QCD reggeon field theory, reggeon interaction vertices are computed as a sum of several contributions. As an example, the triple pomeron vertex is obtained from the transition vertex: $2 \rightarrow 4$ reggeized gluons. This interaction vertex is the sum of the integral kernel $K_{2 \rightarrow 4}$ plus also other 'induced pieces' which, at the end, leads to the conformal invariant expression $V_{4 \leftarrow 2}$. The derivation of this vertex was an outcome of the calculation of the high energy behavior of the six point function (more precisely: 


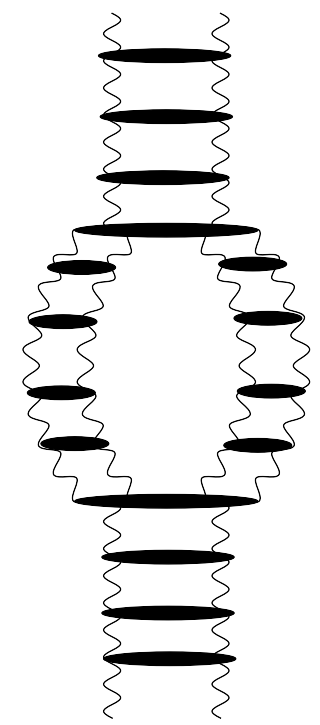

Figure 3. Pomeron self-mass.

from energy discontinuities of the corresponding scattering amplitude). This six point amplitude contains the triple pomeron vertex, as the single splitting of a pomeron in two, illustrated in figure 1. The obtained result is valid in all orders $N_{c}$, and the equivalence, in the large- $N_{c}$ limit, with (1.1) was proven in [7].

In order to catch up with the fan diagrams of the BK-equation, which have been derived in the color dipole approach, one needs to consider more than one consecutive splitting: the simplest example is shown figure 2. Within QCD reggeon field theory, this requires the study of amplitudes containing al least 6 reggeized gluons at the lower end, i.e. the scattering of a projectile on three color singlet targets. In earlier demonstrations [14] it was tacitly assumed that in consecutive splittings exactly the same vertices $\Gamma$ appear. An explicit derivation, however, is still missing. Also, it will be interesting to what extent the fan structure is complete and will be valid also beyond the large- $N_{c}$ limit. It is the purpose of this study to fill some of these gaps.

An investigation of amplitudes with up to six reggeized gluons at the lower end has been presented in [1]. The results, in principle, allow to find, in the leading-log approximation, the complete structure of the evolution of the 2,4, and 6 gluon systems. However, the final step of analyzing the transition $6 \rightarrow 4$ gluons has not been completed. In the present paper we apply the large- $N_{c}$ limit and show that, in fact, the fan structure with two consecutive triple pomeron vertices appears, in full agreement with the BK equations. There are, however, two features which do not affect the validity of the fan structure but go beyond the well-known results. First, we find additional contributions related to the reggization of the gluon. We will dicuss their potential significance. Next, our derivation includes the vertex: pomeron $\rightarrow$ two odderons [1]. As we will discuss in more detail, this vertex is slightly more general than the one used in extensions of the BK equation $[15,16]$.

Our paper is organized as follows. We first (section 2) recapitulate the main steps of the derivation of the triple pomeron vertex in the context of the six point scattering amplitude 


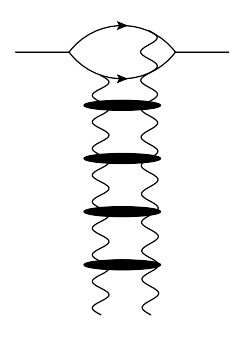

a

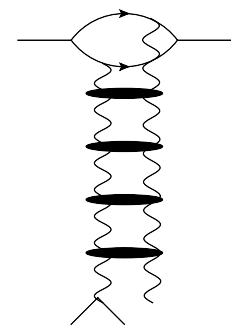

$b$

Figure 4. (a) the amplitudes $D_{2}$ and (b) $D_{3}$.

(i.e. four gluons at the lower end). We will restrict ourselves to the most essential steps and, for details, refer to the literature. We then (section 3) summarize the main results for the eight point reggeon amplitude (i.e.six gluons at the lower end) obtained in [1]. In section 4 we concentrate on the large- $N_{c}$ limit and derive the fan structure where the same triple pomeron vertex describes the consecutive splitting of two BFKL pomerons. In addition, we find reggeizing pieces and discuss their potential significance. In a summarizing section we discuss our results in comparison with the BK equation.

\section{The $2 \rightarrow 4$ reggeized gluon transition: the triple-pomeron vertex $V_{4}$}

To formulate the problem and to fix our notations we first recall the main steps of the derivation of the triple -pomeron vertex $V_{4 \leftarrow 2}$ in QCD reggeon field theory, the theory of interacting reggeized gluons. It will be useful to recapitulate both the original derivation [2] (which is valid in all orders $\left.N_{c}\right)$ and the large- $N_{c}\left(N_{c} \gg 1\right)$ derivation in [17] which makes use of the cylinder topology of the pomeron.

\subsection{Two and three gluon states coupled to the $q \bar{q}$ loop}

The structure of intermediate states coupled to the upper fermion loop in figure 1 has long been known [17]. We begin with the simplest case, $D_{2}$ in figure $4 \mathrm{a}$, which describes a single BFKL pomeron coupled to the fermion loop. The BFKL pomeron is a bound state of two reggeized gluons, and $D_{2}$ satisfies the equation

$$
S_{2} D_{2}=D_{20}+V_{12} D_{2} .
$$

Here $S_{2}$ is the two-reggeon free Schroedinger operator for energy $j_{1}=1-j$

$$
S_{2}=j_{1}+\omega(1)+\omega(2),
$$

where $j$ is the angular momentum, $\omega(i)$ the reggeon Regge trajectory with momentum $q_{i}$, and $V_{12}$ the BFKL kernel written as an operator in the transverse momentum space (with the factor $g^{2} N_{c}$ included). Both $\omega(i)$ and $V_{12}$ are well known and can be found in the literature. $D_{20}(1,2) \equiv D_{20}\left(q_{1}, q_{2}\right)$ is the momentum dependent part of the impact factor describing the four different couplings of the two reggeons 1 and 2 to the fermion loop. With the color dependence included we have

$$
D_{20}^{a_{1} a_{2}}(1,2)=\delta_{a_{1} a_{2}} D_{20}(1,2) .
$$


$D_{2}$ denotes the full BFKL pomeron coupled to the impact factor (including angular momentum $j$ and both color and transverse momentum).

Here and in the following we adopt, for simplicity, the following notation: the product of two operators includes the integration over transverse momenta, e.g.

$$
V_{12} D_{2}:=\frac{g^{2} N_{c}}{8 \pi^{2}} \int d^{2} k V_{12}\left(k_{1}, k_{2} \mid k_{1}+k, k_{2}-k\right) D_{2}\left(k_{1}+k, k_{2}-k\right) .
$$

Note that we define the operator $V_{12}$ in such a way that includes the r.h.s. momentum propagators $\frac{1}{\left(k_{1}+k\right)^{2}\left(k_{2}-k\right)^{2}}$.

Formally we can solve (2.1) by introducing the BFKL Green's-function $G_{2}$ which describes the infinite sum of ladder graphs. Sometimes it is convenient to use, instead of angular momentum $j$, rapidity $y$. We put

$$
S_{2}=\partial_{y}+\omega(1)+\omega(2)
$$

and obtain the rapidity dependent Green's function $G_{2}(y)$. We then can write the solution as a function of $y$ :

$$
D_{2}(y)=G_{2}(y) D_{20},
$$

where $G_{2}(y)$ satisfies the equation

$$
\left(S_{2}-V_{12}\right) G_{2}(y)=\delta(y) .
$$

Now consider the coupling of three reggeized gluons to the fermion loop. It is important to note that the three gluons at the lower end may either come from a direct coupling the fermion loop or from transitions from two reggeons coupled to the loop, which afterwards transform into three reggeons by means of the splitting kernel $K_{3 \leftarrow 2} \cdot{ }^{2}$ As a result, the equation for the corresponding amplitude $D_{3}$ contains two inhomogeneous terms:

$$
S_{3} D_{3}=D_{30}+D_{3 \leftarrow 2}+\frac{1}{2}\left(V_{12}+V_{23}+V_{31}\right) D_{3} .
$$

Here $D_{30}$ denotes the fermion loop with three reggeons coupled to it in all possible ways. Explicitly

$$
D_{30}^{a_{1} a_{2} a_{3}}(1,2,3)=-\frac{1}{2} f^{a_{1} a_{2} a_{3}} g\left(D_{20}(2,31)-D_{20}(1,23)-D_{20}(3,12)\right) .
$$

Here ' 12 ' is our short-hand notation for the sum of the two momenta $q_{1}+q_{2}$. The transition from 2 to 3 reggeons, $D_{3 \leftarrow 2}$, is accomplished by the kernel $K_{3 \leftarrow 2}$

$$
D_{3 \leftarrow 2}(y)=K_{3 \leftarrow 2} D_{2}(y)=K_{3 \leftarrow 2} G_{2}(y) D_{20},
$$

where the momentum dependent part of the $2 \rightarrow 3$ kernel (including the momentum propagators for the intermediate two reggeon state) has the form:

$$
K_{3 \leftarrow 2}\left(k_{1}, k_{2}, k_{3} \mid q_{1}, q_{2}\right)=g^{3} N_{c}\left\{\frac{q_{12}^{2}}{q_{1}^{2} q_{2}^{2}}+\frac{k_{2}^{2}}{\left(q_{1}-k_{1}\right)^{2}\left(q_{2}-k_{3}\right)^{2}}-\frac{k_{12}^{2}}{q_{1}^{2}\left(q_{2}-k_{3}\right)^{2}}-\frac{k_{23}^{2}}{q_{2}^{2}\left(q_{1}-k_{1}\right)^{2}}\right\} .
$$

\footnotetext{
${ }^{2}$ Throughout this paper we find it convenient to orient, in the subscripts of the operators, the arrows from right to left. This is consistent with reading our equations which contain products of operators (beginning with (2.1)) from right to left. In our figures, this corresponds to moving down from top to bottom.
} 
It is remarkable that the solution of the equation (2.8) is quite simple, and can totally expressed via the pomeron wave function $D_{2}$ [2] (figure $4(\mathrm{~b})$ ):

$$
D_{3}^{a_{1} a_{2} a_{3}}(1,2,3)=-\frac{1}{2} g f^{a_{1} a_{2} a_{3}} g\left(D_{2}(2,31)-D_{2}(1,23)-D_{2}(12,3)\right) .
$$

This solution has the same momentum structure as the one-loop impact factor (2.9). Both (2.12) and (2.9) have the cylinder topology.

The three terms on the r.h.s. of (2.12) have a simple interpretation. As an example, the third term, $D_{2}(12,3)$ illustrated in figure $4(\mathrm{~b})$ ), describes a single BFKL pomeron, where the left constituent reggeon carries the sum of two momenta, $q_{1}+q_{2}$, whereas the constituent reggeon on the r.h.s. carries the momentum $q_{3}$. If the $q_{i}$ refer to targets $1,2,3$ to which the pomeron couples, the left reggeon splits into two gluons which then couple to target 1 and target 2 . Here it is important to note the difference between wavy lines (which correspond to reggeized gluons) and straight lines (which denote elementary gluons): the former ones contain evolution in rapidity whereas the latter ones do not. Figure 4(b)) thus illustrates the nature of reggeization: a reggeized gluon can be viewed as a bound state of two elementary gluons. Later on we will see that a reggeized can also be viewed as a bound state of three or even more gluons.

\subsection{The triple pomeron vertex}

We now extend the procedure to transitions into four reggeized gluons. Let us first follow the derivation described in [2]. The coupling of four reggeized gluons to the fermion loop consists of two groups:

$$
\begin{aligned}
D_{40}^{a_{1} a_{2} a_{3} a_{4}}(1,2,3,4)= & -g^{2} d^{a_{1} a_{2} a_{3} a_{4}}\left(D_{20}(123,4)+D_{20}(1,234)-D_{20}(14,23)\right) \\
& -g^{2} d^{a_{2} a_{1} a_{3} a_{4}}\left(D_{20}(134,2)+D_{20}(124,3)-D_{20}(12,34)-D_{20}(13,24)\right),
\end{aligned}
$$

where the color tensor is defined as

$$
\begin{aligned}
d^{a_{1} a_{2} a_{3} a_{4}} & =\operatorname{Tr}\left(t^{a_{1}} t^{a_{2}} t^{a_{3}} t^{a_{4}}+t^{a_{4}} t^{a_{3}} t^{a_{2}} t^{a_{1}}\right) . \\
& =\frac{1}{2 N_{c}} \delta_{a_{1} a_{2}} \delta_{a_{3} a_{4}}+\frac{1}{4}\left(d_{a_{1} a_{2} c} d_{c a_{3} a_{4}}+f_{a_{1} a_{2} c} f_{c a_{3} a_{4}}\right) .
\end{aligned}
$$

By drawing all terms on the surface of a cylinder, the two groups differ in the order of $t$-channel gluons. The integral equation for $D_{4}$ can be written as

$$
S_{4} D_{4}=D_{4 ; 0}+\sum K_{3 \leftarrow 2} D_{3}+K_{4 \leftarrow 2} D_{2}+\sum_{(i j)} V_{i j} D_{4},
$$

where the momentum dependent part of the kernel $K_{4 \leftarrow 2}$ has the form:

$$
\begin{aligned}
K_{4 \leftarrow 2}\left(k_{1}, k_{2}, k_{3}, k_{4} \mid q_{1}, q_{2}\right) & =g K_{3 \leftarrow 2}\left(k_{1}, k_{23}, k_{4} \mid q_{1}, q_{2}\right) \\
& =g^{4} N_{c}\left\{\frac{q_{12}^{2}}{q_{1}^{2} q_{2}^{2}}+\frac{\left(k_{2}+k_{3}\right)^{2}}{\left(q_{1}-k_{1}\right)^{2}\left(q_{2}-k_{4}\right)^{2}}-\frac{k_{123}^{2}}{q_{1}^{2}\left(q_{2}-k_{4}\right)^{2}}-\frac{k_{234}^{2}}{q_{2}^{2}\left(q_{1}-k_{1}\right)^{2}}\right\} .
\end{aligned}
$$



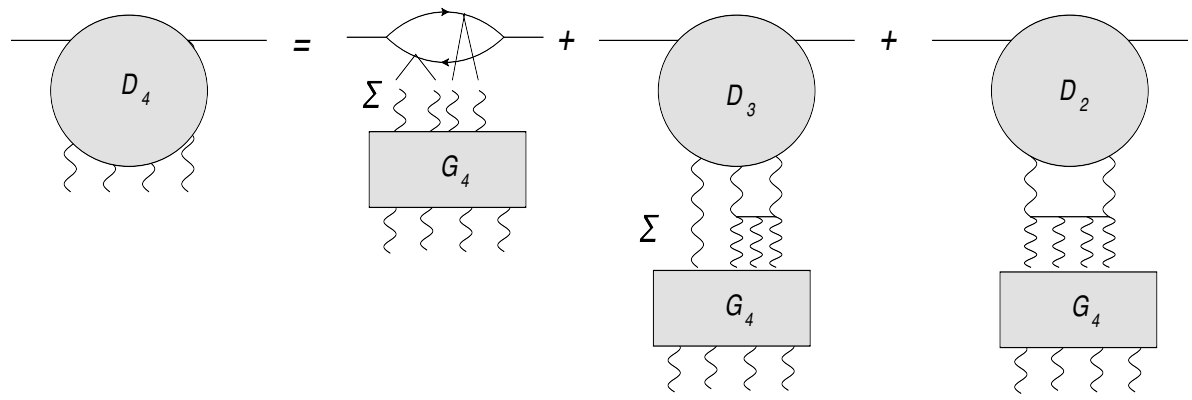

Figure 5. Amplitude with 4 reggeized gluons. $G_{4}$ denotes the Green's function for 4 reggeized gluons.

Formally we could solve (2.15) by

$$
D_{4}(y)=G_{4}(y) D_{40}+\int d y^{\prime} G_{4}\left(y-y^{\prime}\right)\left[\sum K_{3 \leftarrow 2} D_{3}\left(y^{\prime}\right)+K_{4 \leftarrow 2} D_{2}\left(y^{\prime}\right)\right] .
$$

This solution is illustrated in figure 5 .

However, this is not the final solution. Instead, one first removes a reggeized piece with only two reggeized gluons, i.e. one makes the ansatz (figure 6)

$$
D_{4}=D_{4}^{R}+D_{4}^{I}
$$

with

$$
\begin{aligned}
D_{4}^{R ; a_{1} a_{2} a_{3} a_{r}}(1,2,3,4)= & -g^{2} d^{a_{1} a_{2} a_{3} a_{4}}\left(D_{2}(123,4)+D_{2}(1,234)-D_{2}(14,23)\right) \\
& -g^{2} d^{a_{2} a_{1} a_{3} a_{4}}\left(D_{2}(134,2)+D_{2}(124,3)-D_{2}(12,34)-D_{2}(13,24)\right) .
\end{aligned}
$$

Inserting this into (2.15) and using the integral equation for $D_{2}$, eq. (2.1), we combine all terms containing $D_{2}$ and arrive at the equation for $D_{4}^{I}$

$$
S_{4} D_{4}^{I}=V_{4 \leftarrow 2} D_{2}+\sum_{(i j)} V_{i j} D_{4}^{I}
$$

with the solution

$$
D_{4}^{I}(y)=\int d y^{\prime} G_{4}\left(y-y^{\prime}\right) V_{4 \leftarrow 2} D_{2}\left(y^{\prime}\right) .
$$

Here $V_{4 \leftarrow 2}$ denotes the triple pomeron vertex as an operator in the color and transverse momentum space. Exhibiting the color structure, this vertex can be written as a sum of three terms:

$$
\begin{aligned}
\left.V_{4 \leftarrow 2}^{a_{1} a_{2} a_{3} a_{4}}\left(k_{1} k_{2} k_{3} k_{4} \mid q_{1}, q_{2}\right)\right)= & \delta_{a_{1} a_{2}} \delta_{a_{3} a_{4}} V_{4}(1,2 ; 3,4)+\delta_{a_{1} a_{3}} \delta_{a_{2} a_{4}} V_{4}(1,3 ; 2,4) \\
& +\delta_{a_{1} a_{4}} \delta_{a_{2} a_{3}} V_{4}(1,4 ; 2,3)
\end{aligned}
$$

where on the r.h.s. we have suppressed the initial momenta $q_{1}, q_{2}$ which in the convolution with $D_{2}$ are to be integrated, and we have written only the momentum structure of the two 

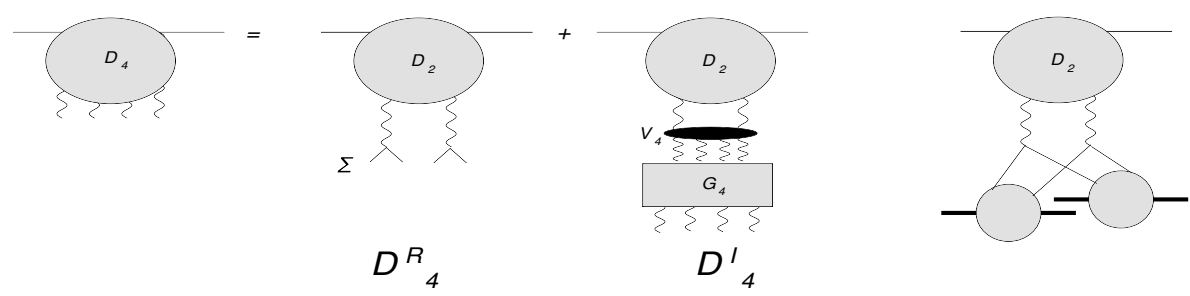

Figure 6. (a) Amplitude with 4 reggeized gluons: decomposition into reggeized (left) and irreducible (right) pieces. The detailed form of the reggeized part, $D_{2}$, is given in 2.19). It is the just the BFKL pomeron where each lower leg carries sums of momenta and finally splits into elementary gluons. (b) Example of the coupling of a reggeized term to two nucleons.

outgoing pomerons with the substitutions $k_{i} \rightarrow i,(i=1, \ldots, 4)$. The function $V_{4}(1,2 ; 3,4)$ is symmetric under permuting $(1,2)$ or $(3,4)$, and under the exchange of the pairs $(1,2)$ and (34); also, the convolution $V_{4}(1,2 ; 3,4) D_{2}$ vanishes whenever one of the four transverse momenta goes to zero. For the full vertex one observes Bose symmetry under the exchange of reggeized gluons 1,2,3,4. Considering the configuration where the pairs $(1,2)$ and $(34)$ are color singlet and taking the large- $N_{c}$ limit we find that in (2.22) only the first term contributes, and integral equation for $D_{4}^{I}$ simpifies:

$$
S_{4} D_{4}^{I}((1,2),(3,4))=\left(V_{4} D_{2}\right)(12,34)+\left(V_{12}+V_{34}\right) D_{4}^{I},
$$

where we have indicated that, in both color and momentum, the arguments of $V_{4} D_{2}$ and $D_{2}$ are grouped into the pairs $(1,2)$ and $(3,4)$. Since the rapidity evolution of these pairs proceeds independently, the Green's function $G_{4}$ factorizes:

$$
G_{4}(y ; 1234) \rightarrow G_{2}(y ; 12) G_{2}(y ; 34),
$$

and $D_{4}^{I}$ depends upon two independent rapidity variables, $y_{1}$ and $y_{2}$ :

$$
D_{4}^{I}\left(y_{1}, y_{2} ; 12,34\right)=\int d y^{\prime} G_{2}\left(y_{1}-y^{\prime} ; 12\right) G_{2}\left(y_{2}-y^{\prime} ; 34\right)\left(V_{4} D_{2}\left(y^{\prime}\right)\right) .
$$

After Fourier transformation to coordinate space, this expression takes the form (1.1) (with suitable substitutions for the three pomerons). A general proof (for general $N_{c}$ ) of this Fourier tansform, has been presented in [7].

Finally we make a few comments on the reggeizing term $D_{4}^{R}$. As indicated in figure 6a. and described in (2.19), the reggeized term comes as a sum of single BFKL pomerons where the lower legs of reggeized gluons split into four elementary gluons. $D_{4}^{R}$ represents an additive correction to the single pomeron in figure 4a with two gluons at the lower end. In the scattering on a big nucleus the pomeron in figure $4 \mathrm{a}$, in its simplest form, couples to a single nucleon inside the nucleus, and this coupling can be described by the gluon distribution of the nucleon. Following this simple picture, the corrections contained in $D_{4}^{R}$ then can be interpreted as the coupling of a single BFKL pomeron to a pair of 
two nucleons. As an example, consider the last term in (2.19), $D_{2}(13,24)$ (remember that we are considering the configuration where the pairs (12) and (34) are in color singlet states). As illustrated in figure 6(b), in this contribution the BFKL pomeron couples to two different nucleons. Since all the rapidity evolution is contained in the reggeized gluons, this coupling to two nucleons is a 'low energy' interaction. As we will discuss in section 4.4 , this new contribution can be interpreted as the second order term in an eikonal initial condition for the nonlinear evolution equations [18].

\subsection{The topological derivation}

As observed in [17], the large- $N_{c}$ limit of the triple pomeron vertex can be obtained also in another way, by making use of the cylinder topology of the pomeron in the large- $N_{c}$ approximation.

To this end we return to $D_{40}$ in (2.13) and note that the two groups correspond to two different configurations on the surface of the cylinder, $D_{4}^{1234}$ and $D_{4}^{2134}$ :

$$
D_{40}^{a_{1} a_{2} a_{3} a_{4}}(1,2,3,4)=-d^{a_{1} a_{2} a_{3} a_{4}} D_{40}^{1234}(1,2,3,4)-d^{a_{2} a_{1} a_{3} a_{4}} D_{40}^{2134}(1,2,3,4),
$$

where

$$
\begin{aligned}
& D_{40}^{1234}=g^{2}\left(D_{20}(1,234)+D_{20}(123,4)-D_{20}(14,23)\right) \\
& D_{40}^{2134}=g^{2}\left(D_{20}(2,134)+D_{20}(124,3)-D_{20}(12,34)-D_{20}(13,24)\right) .
\end{aligned}
$$

They differ in the order of gluons on the surface of a cylinder (indicated by the upper index), and they do not mix. The evolution in rapidity of the four-reggeon-state can start directly from the fermion loop, as described in (2.27) and (2.28), or from two- and threereggeon states with subsequent transitions to a four gluon state. One finds that transitions from the two-reggeon states into $D_{4}^{2134}$ are prohibited. So the equations are

$$
S_{4} D_{4}^{1234}=D_{40}^{1234}+D_{4 \leftarrow 2}^{1234}+D_{4 \leftarrow 3}^{1234}+\frac{1}{2}\left(V_{12}+V_{23}+V_{34}+V_{41}\right) D_{4}^{1234}
$$

and

$$
S_{4} D_{4}^{2134}=D_{40}^{2134}+D_{4 \leftarrow 3}^{2134}+\frac{1}{2}\left(V_{21}+V_{13}+V_{34}+V_{42}\right) D_{4}^{2134} .
$$

Here the transition from 2 to 4 reggeons is described by

$$
D_{4 \leftarrow 2}^{1234}=-K_{4 \leftarrow 2} D_{2} .
$$

For the transition $3 \rightarrow 4$ we have:

$$
\begin{aligned}
D_{4 \leftarrow 3}= & \frac{1}{2}\left(K_{3 \leftarrow 2}\left(1,2,3 \mid 1^{\prime} 3^{\prime}\right) D_{3}\left(1^{\prime}, 3^{\prime}, 4\right)-K_{3 \leftarrow 2}\left(1,2,4 \mid 1^{\prime}, 4^{\prime}\right) D_{3}\left(1^{\prime}, 3,4^{\prime}\right)\right. \\
& \left.+K_{3 \leftarrow 2}\left(2,3,4 \mid 2^{\prime}, 4^{\prime}\right) D_{3}\left(1,2^{\prime}, 4^{\prime}\right)-K\left(1,3,4 \mid 1^{\prime}, 4^{\prime}\right) D_{3}\left(1^{\prime}, 2,4^{\prime}\right)\right),
\end{aligned}
$$

where $D_{3}$ is the solution of eq. (2.8). As shown in [17], eqs. (2.29) and (2.30) for $D_{4}$ can be solved directly: the solutions can be obtained from (2.27) and (2.28) by substituting $D_{20} \rightarrow D_{2}$. Their sum coincides with $D_{4}^{R}$ in (2.19). 
In the next step we consider another toplogical configuration, which is suppressed in $1 / N_{c}$ : the formation of two color singlet cylinders made of reggeons pairs $(1,2)$ and $(3,4)$ : we denote this amplitude by $D_{4}^{2 P}$. These two cylinders can start directly from the fermion loop or from a single cylinder formed by 2, 3 or 4 reggeon which then couples to two cylinders. The full amplitude $D_{4}^{2 P}$ satisfies the equation [17]:

$$
S_{4} D_{4}^{2 P}=D_{2 P 0}+D_{2 P \leftarrow 2}+D_{2 P \leftarrow 3}+D_{2 P \leftarrow 4}+\left(V_{12}+V_{34}\right) D_{4}^{2 P} .
$$

The inhomogeneous term $D_{2 P 0}$ is just the projection of the loop $D_{40}$ onto the 2pomeron state:

$$
D_{2 P 0}=\frac{1}{2} N_{c}\left(D_{40}^{1234}+D_{40}^{2134}\right) .
$$

The inhomogeneous terms $D_{4 \leftarrow 2}^{2 P}, D_{4 \leftarrow 3}^{2 P}$ and $D_{4 \leftarrow 4}^{2 P}$ describe the mentioned transitions into the two-pomeron state from two, three and four evolved reggeons coming from the loop.

$$
D_{2 P \leftarrow 2}=-K_{4 \leftarrow 2} D_{2},
$$

where

$$
K_{4 \leftarrow 2}\left(1,2,3,4 \mid 1^{\prime}, 2^{\prime}\right)=g K_{3 \leftarrow 2}\left(1,23,4 \mid 1^{\prime}, 2^{\prime}\right),
$$

and $D_{2}$ is the solution of $(2.1)$.

$$
\begin{aligned}
D_{2 P \leftarrow 3}= & \frac{1}{2}\left(K_{3 \leftarrow 2}\left(1,2,3 \mid 1^{\prime} 3^{\prime}\right) D_{3}\left(1^{\prime}, 3^{\prime}, 4\right)-K_{3 \rightarrow 2}\left(1,2,4 \mid 1^{\prime}, 4^{\prime}\right) D_{3}\left(1^{\prime}, 3,4^{\prime}\right)\right. \\
& \left.+K_{3 \leftarrow 2}\left(2,3,4 \mid 2^{\prime}, 4^{\prime}\right) D_{3}\left(1.2^{\prime}, 4^{\prime}\right)-K\left(1,3,4 \mid 1^{\prime}, 4^{\prime}\right) D_{3}\left(1^{\prime}, 2,4^{\prime}\right)\right),
\end{aligned}
$$

where $D_{3}$ is the solution of eq. (2.8). The transition from the single cylinder to the twocylinder configuration is described by

$$
D_{2 P \leftarrow 4}=\left(V_{23}+V_{14}-V_{23}-V_{24}\right)\left(D_{4}^{1234}-D_{4}^{2134}\right),
$$

where $D_{4}^{1234}$ and $D_{4}^{2134}$ are the solutions to eqs. (2.29) and (2.30). In order to solve (2.33) we collect all terms containing $D_{2}$ and arrive at (2.23) with the same triple pomeron vertex function $V_{4}$. Formally we can solve this equation:

$$
D_{4}^{2 P}(y)=\int_{0}^{y} d y_{1} d y_{2} \delta\left(y_{1}+y_{2}-y\right) G_{2 P}\left(y_{2}\right) V_{4} D_{2}\left(y_{1}\right),
$$

where $G_{2 P}=\left(G_{2}\right)^{2}$ is the Green function for two non-interacting pomerons. For the complete four reggeon amplitude we still have to add the single cylinder contribution $D_{4}^{R}$.

We finally want to stress that all results obtained so far do not use the explicit form of function $D_{20}(1,2)$, and they remain valid if one substitutes $D_{20}$ by an arbitrary function $F_{2}(1,2)$ with properties

$$
F_{2}\left(q_{1}, q_{2}\right)=F_{2}\left(q_{2}, q_{1}\right), \quad F_{2}\left(0, q_{2}\right)=F_{2}\left(q_{1}, 0\right)=0 .
$$

All one needs is that the three and four-reggeon contributions come from the impact factors, $F_{3}$ and $F_{4}$, related to $F_{2}$ in exactly the same manner as $D_{30}$ and $D_{40}$ are related to $D_{20}$ in eqs. (2.9) and (2.13), namely

$$
F_{3}^{a_{1} a_{2} a_{3}}(1,2,3)=-\frac{1}{2} f^{a_{1} a_{2} a_{3}} g\left(F_{2}(2,31)-F_{2}(1,23)-F_{2}(3,12)\right)
$$


and

$$
\begin{aligned}
F_{4}^{a_{1} a_{2} a_{3} a_{4}}(1,2,3,4)= & -g^{2}\left\{d^{a_{1} a_{2} a_{3} a_{4}}\left(F_{2}(1,234)+F_{2}(123,4)-F(14,23)\right)\right. \\
& \left.+d^{a_{2} a_{1} a_{3} a_{4}}\left(F_{2}(2,134)+F_{2}(124,3)-F_{2}(12,34)-F_{2}(13,24)\right)\right\} .
\end{aligned}
$$

\section{The transition $2 \rightarrow 6$}

Let us now turn to the amplitudes with five and six reggeized gluons at the lower end. For the time being we postpone the large- $N_{c}$ limit and keep the number of colors finite. In [1] an integral equation for $D_{6}$ has been derived and analyzed. The final step, however, the analysis of the irreducible piece $D_{6}^{I}$, has not been completed. In section 4 we return to the large- $N_{c}$ limit, and we will show that in this limit $D_{6}^{I}$, contains the fan diagrams entering the Balitsky-Kovchegov (BK) equation.

Let us first review the results obtained in [1]. The structure of the integral equation is illustrated in figure 7. The analysis described in the previous section has illustrated that the functions $D_{i}$ exhibit the following hierarchy structure: $D_{3}$ is built from $D_{2}, D_{4}$ is based upon $D_{2}$ and $D_{3}$. Similarly, $D_{5}$ contains $D_{2}, D_{3}$, and $D_{4}$, and $D_{6}$ is obtained from all $D_{i}$ with $i<6$. This is illustrated by the integral equation which, in a somewhat simplified form, reads as follows:

$$
S_{6} D_{6}=D_{60}+K_{6 \leftarrow 2} D_{2}+\sum K_{5 \leftarrow 2} D_{3}+\sum K_{4 \leftarrow 2} D_{4}+\sum K_{3 \leftarrow 2} D_{5}+\sum V_{i j} D_{6}
$$

with

$$
\begin{aligned}
& D_{60}^{a_{1} a_{2} a_{3} a_{4} a_{5} a_{6}}(1,2,3,4,5,6)= \\
& \quad g^{6}\left\{d^{a_{1} a_{2} a_{3} a_{4} a_{5} a_{6}}\left[D_{20}(12345,6)+D_{20}(1,23456)-D_{20}(16,2345)\right]\right. \\
& \quad+d^{a_{2} a_{1} a_{3} a_{4} a_{5} a_{6}}\left[D_{20}(1345,62)-D_{20}(1345,62)+D_{20}(126,345)-D_{20}(12,3456)\right] \\
& \quad+d^{a_{1} a_{2} a_{3} a_{4} a_{6} a_{5}}\left[D_{20}(12346,5)-D_{20}(1234,56)+D_{20}(156,234)-D_{20}(15,2346)\right] \\
& \quad+d^{a_{2} a_{1} a_{3} a_{4} a_{6} a_{5}}\left[-D_{20}(1256,34)-D_{20}(1346,25)+D_{20}(125,346)+D_{20}(134,256)\right] \\
& \quad+d^{a_{3} a_{1} a_{2} a_{4} a_{5} a_{6}}\left[D_{20}(12456,3)-D_{20}(1245,36)+D_{20}(136,245)-D_{20}(13,2456)\right] \\
& \quad+d^{a_{1} a_{2} a_{3} a_{5} a_{6} a_{4}}\left[D_{20}(12356,4)-D_{20}(1235,46)+D_{20}(146,235)-D_{20}(14,2356)\right] \\
& \quad+d^{a_{2} a_{1} a_{3} a_{5} a_{6} a_{4}}\left[-D_{20}(1246,35)-D_{20}(1356,24)+D_{20}(124,356)+D_{20}(135,246)\right] \\
& \left.\quad+d^{a_{1} a_{2} a_{3} a_{6} a_{5} a_{4}}\left[-D_{20}(1236,45)-D_{20}(1456,23)+D_{20}(123,456)+D_{20}(145,236)\right]\right\},
\end{aligned}
$$

where

$$
d^{a_{1} a_{2} a_{3} a_{4} a_{5} a_{6}}=\operatorname{Tr}\left(t^{a_{1}} t^{a_{2}} t^{a_{3}} t^{a_{4}} t^{a_{5}} t^{a_{6}}\right)+\operatorname{Tr}\left(t^{a_{6}} t^{a_{5}} t^{a_{4}} t^{a_{3}} t^{a_{2}} t^{a_{1}}\right) .
$$


One easily verifies that all configurations can be drawn on the surface of a cylinder, and they represent the different orderings of the $t$-channel gluon lines.

On the r.h.s. of (3.1), a new ingredient appears, $D_{5}$, for which the following decomposition has been found:

$$
D_{5}=D_{5}^{R}+D_{5}^{I}
$$

where

$$
\begin{aligned}
& D_{5}^{R a_{1} a_{2} a_{3} a_{4} a_{5}}(1,2,3,4,5)= \\
& -g^{3}\left\{f^{a_{1} a_{2} a_{3} a_{4} a_{5}}\left[D_{2}(1234,5)+D_{2}(1,2345)-D_{2}(15,234)\right]\right. \\
& \quad+f^{a_{2} a_{1} a_{3} a_{4} a_{5}}\left[D_{2}(1345,2)-D_{2}(12,345)+D_{2}(125,34)-D_{2}(134,25)\right] \\
& \quad+f^{a_{1} a_{2} a_{3} a_{5} a_{4}}\left[D_{2}(1235,4)-D_{2}(14,235)+D_{2}(145,23)-D_{2}(123,45)\right] \\
& \left.\quad+f^{a_{1} a_{2} a_{4} a_{5} a_{3}}\left[D_{2}(1245,3)-D_{2}(13,245)+D_{2}(135,24)-D_{2}(124,35)\right]\right\}
\end{aligned}
$$

with

$$
f^{a b c d e}=\frac{1}{i}\left[\operatorname{Tr}\left(t^{a} t^{b} t^{c} t^{d} t^{e}\right)-\operatorname{Tr}\left(t^{e} t^{d} t^{c} t^{b} t^{a}\right)\right]
$$

and

$$
\begin{aligned}
& D_{5}^{I a_{1} a_{2} a_{3} a_{4} a_{5}}(1,2,3,4,5)= \\
& \frac{g}{2}\left[f^{a_{1} a_{2} c} D_{4}^{I c a_{3} a_{4} a_{5}}(12,3,4,5)+f^{a_{1} a_{3} c} D_{4}^{I c a_{2} a_{4} a_{5}}(13,2,4,5)\right. \\
& \quad+f^{a_{1} a_{4} c} D_{4}^{I c a_{2} a_{3} a_{5}}(14,2,3,5)+f^{a_{1} a_{5} c} D_{4}^{I c a_{2} a_{3} a_{4}}(15,2,3,4) \\
& \quad+f^{a_{2} a_{3} c} D_{4}^{I a_{1} c a_{4} a_{5}}(1,23,4,5)+f^{a_{2} a_{4} c} D_{4}^{I a_{1} c a_{3} a_{5}}(1,24,3,5) \\
& \quad+f^{a_{2} a_{5} c} D_{4}^{I a_{1} c a_{3} a_{4}}(1,25,3,4)+f^{a_{3} a_{4} c} D_{4}^{I a_{1} a_{2} c a_{5}}(1,2,34,5) \\
& \left.\quad+f^{a_{3} a_{5} c} D_{4}^{I a_{1} a_{2} c a_{4}}(1,2,35,4)+f^{a_{4} a_{5} c} D_{4}^{I a_{1} a_{2} a_{3} c}(1,2,3,45)\right] .
\end{aligned}
$$

We remind that the triple pomeron vertex $V_{4 \leftarrow 2}$ inside $D_{4}^{I a_{1} a_{2} a_{3} a_{4}}$ has the structure described in (2.22).

Turning to the r.h.s. of (3.1) and using our results for $D_{2}, \ldots, D_{5}$ we note that all terms (disregarding, for the moment, the first term $D_{6 ; 0}$ ), have a simple structure of multireggeon states: the second term (containing $D_{3}$ ) and third term (containing $D_{4}$ ) start, at the fermion loop, with a two-reggeon state which then turns into the six-reggeon state. The fourth and fifth terms have both ' $\mathrm{R}$ ' and 'I' contributions; the ' $\mathrm{R}$ '-terms start with two reggeon states and then undergo $2 \rightarrow 6$ transitions. The 'I'-terms begin with a two-reggeon state, then contain the $2 \rightarrow 4$ transition described by the $2 \rightarrow 4$ reggeon vertex discussed in the previous section, and finally end with a transition into the six-reggeon state. This pattern is not altered if we insert, for $D_{6}$, the decomposition

$$
D_{6}=D_{6}^{R}+D_{6}^{I}
$$




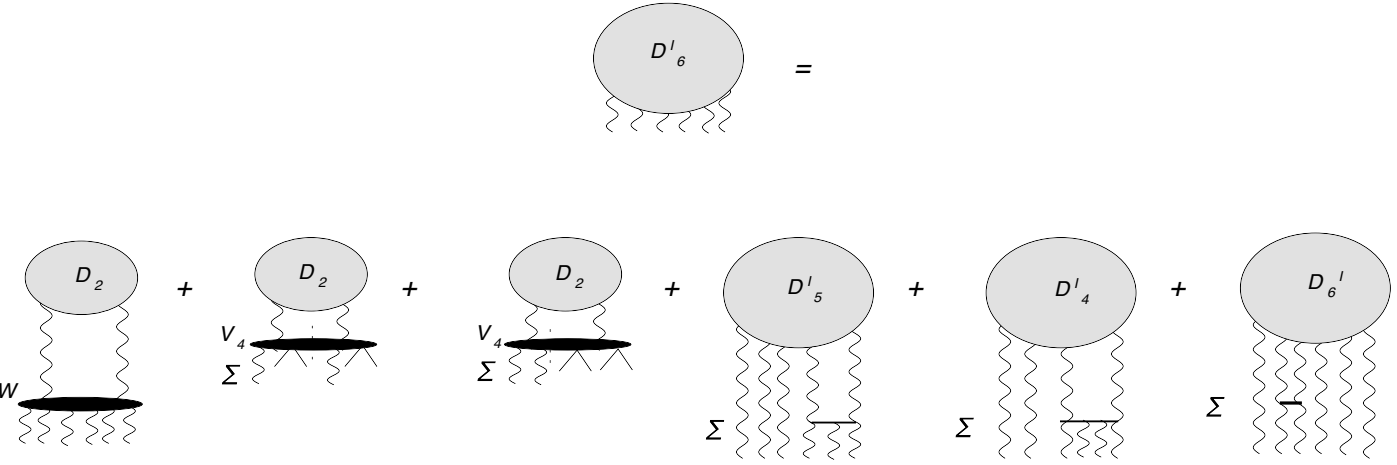

$L$

$1+J$

Figure 7. The integral equation (3.9) for the amplitude $D_{6}^{I}$ with 6 reggeized gluons. In the terms denoted by ' $\mathrm{L}$ ' and ' $\mathrm{I}+\mathrm{J}$ ' the pairs of straight lines attached to the vertices denote reggeons which carry sums of final momenta. For further explanations see text.

with $D_{6}^{R}$ being obtained from $D_{60}$ in (3.2) by replacing on the r.h.s. $D_{20} \rightarrow D_{2}$. Using the BFKL equation for $D_{6}^{R}$ removes, on the r.h.s. of (3.1), the first term, $D_{60}$, and adds new contributions to the transition $2 \rightarrow 6$ vertex. Additional contributions to the $2 \rightarrow 6$ vertex are generated by inserting, in the last term of the r.h.s. of (3.1), $D_{6}^{R}$. The main task therefore is the computation of the complete (and potentially new) $2 \rightarrow 6$ and $4 \rightarrow 6$ vertices. In section 4.4. we will say more about the reggeizing term $D_{6}^{R}$.

A very big step was already done in [1]. Namely, starting from (3.1), inserting (3.8) and performing an extensive recombination of terms a modified equation for $D_{6}^{I}$ was obtained which has the following structure:

$$
S_{6} D_{6}^{I}=W_{\text {odd }} D_{2}+\left(T_{L}+T_{I}+T_{J}\right) D_{2}+\sum K_{3 \leftarrow 2} D_{5}^{I}+\sum K_{4 \leftarrow 2} D_{4}^{I}+\sum V_{i j} D_{6}^{I} .
$$

This equation is illustrated in figure 7 .

Here the first term denotes the $P \rightarrow 2 O$ pomeron-odderon vertex, first derived and discussed in [1]. It has the form:

$$
W_{\text {odd }} D_{2}=\sum_{\text {partitions }} d^{a_{1} a_{2} a_{3}} d^{a_{4} a_{5} a_{6}}\left(W D_{2}\right)(1,2,3 ; 4,5,6),
$$

where the sum extends over all partitions of 6 reggeons into two triplets, and $d^{a b c}$ are the symmetric structure constants of the $\mathrm{SU}(3)$ gauge group. For details of the function $W$ we refer to $[1]$.

Let us say few words about the significance of this vertex. As it is well known, the odderon is a bound state of three reggeized gluons in a C-odd state, and it is natural that the pomeron $\rightarrow$ two-odderon comes as a part of the 2 gluon $\rightarrow 6$ gluon vertex. A special bound state solution of the three gluon system with intercept exactly at one was found in [19]. In this solution, two of the three gluons merge into a single even signature reggeized gluon (degenerate with the 'normal' odd-signature reggeized gluon) and then form a bound state with the third gluon. This results in a quasi-two-gluon bound state 
which is closely related to the BFKL pomeron. For this special odderon solution, the pomeron $\rightarrow$ two-odderon vertex becomes similar to the triple pomeron vertex. It is this special odderon solution which appears in the generalizations of the BK-equation $[15,16]$. Our more general version (3.10), on the other hand, allows to inlcude also other three-gluon solutions, such as the one found in $[20,21]$ with intercept slightly below one.

Returning to the equation (3.9). the second term has the form:

$$
T_{L} D_{2}=\sum_{\text {partitions }} f^{a_{1} a_{2} a_{3}} f^{a_{4} a_{5} a_{6}} L(1,2,3 ; 4,5,6),
$$

where the sum extends over all partitions of 6 reggeons in two groups with three reggeons in each. The function $L$ consists of $3^{2}=9$ terms which, for the partition (123)(456) can be written as follows:

$$
L(1,2,3 ; 4,5,6)=\frac{g^{2}}{4} \sum_{i j k} \sum_{l m n}\left(V_{4} D_{2}\right)(i j, k ; l m, n),
$$

where the sums are defined as follows

$$
\begin{aligned}
& \sum_{i j k}=(12,3)+(1,23)-(13,2) \\
& \sum_{l m n}=(45,6)+(4,56)-(46,5) .
\end{aligned}
$$

Here each sum has a structure similar to $D_{30}$ in (2.9). In the second term of figure 7 , the summation sign denotes both the summation over partitions (3.11) and the summations contained in (3.12). As it can be read off from figure 7, these L-terms contain a transition described by $V_{4 \leftarrow 2}$ : a pair of two reggeized gluons $\rightarrow$ splits into two colorless triplets of reggeized gluons. For each triplet, the initial momentum structure of the L-terms is similar to $D_{30}$ in (2.9). Except for the large- $N_{c}$ limit, where each triplet evolves independently in rapidity, in the course of the subsequent evolution the triplets interactwith each other and thus loose their initial triplet structure.

Next we discuss the third term, which consists of two terms labeled by ' $\mathrm{I}$ ' and ' $\mathrm{J}$ '. We first list the color structure:

$$
T_{I} D_{2}=\sum_{\text {partitions }} \delta_{a_{1} a_{2}} d^{a_{3} a_{4} a_{5} a_{6}} I(1,2 ; 3,4,5,6)
$$

and

$$
T_{J} D_{2}=\sum_{\text {partitions }} \delta_{a_{1} a_{2}} d^{a_{4} a_{3} a_{5} a_{6}} J(1,2 ; 3,4,5,6) .
$$

In both terms we sum over all partitions of 6 reggeons into two groups with 4 reggeons in one and 2 in the other one. In detail, for the partition (12)(3456):

$$
I(1,2 ; 3,4,5,6)=-g^{2}\left(\left(V_{4} D_{2}\right)(1,2 ; 3,456)+\left(V_{4} D_{2}\right)(1,2 ; 345,6)-\left(V_{4} D_{2}\right)(1,2 ; 36,45)\right)
$$


and

$$
\begin{array}{r}
J(1,2 ; 3,4,5,6)=-g^{2}\left(\left(V_{4} D_{2}\right)(1,2 ; 356,4)+\left(V_{4} D_{2}\right)(1,2 ; 346,5)\right. \\
\left.-\left(V_{4} D_{2}\right)(1,2 ; 34,56)-\left(V_{4} D_{2}\right)(1,2 ; 35,46)\right) .
\end{array}
$$

Focussing on the subsystem (3456) and comparing the function $I$ with the first line of $D_{40}$ in (2.13) one sees the identical structure in the arguments; similarly, the four terms in the function $J$ coincide with the second line in (2.13). Following our discussion of the ' $L$ '-term, we interpret this structure as a transition: a colorless pair of reggeized gluons $\rightarrow$ a colorless pair + a colorless quartet of reggeized gluons. Initially, at the vertex $V_{4 \leftarrow 2}$, the quartet has the same momentum structure as $D_{40}$ in (2.13). During the subsequent evolution the separation of pair+quartet will be lost.

Finally, the last two terms in figure 7 still contain 'elementary kernels', $K_{3 \leftarrow 2}$ and $K_{4 \leftarrow 2}$ which require further recombination. In the terms with $K_{3 \leftarrow 2}$ and $K_{4 \leftarrow 2}$ the sum extends over all pairs of gluons inside $D_{5}^{I}$ and $D_{4}^{I}$, resp.

As we have said before, for general $N_{c}$ the missing next step has not been carried out. In the following we restrict ourselves to the large- $N_{c}$ limit and describe the solution of eq. (3.9) (and figure 7).

\section{The large- $N_{c}$ limit of $D_{6}$}

\subsection{New reggeizing pieces}

As already indicated in [1], the integral equation (3.9) does not represent the final version. Instead, it will be necessary, once more, to separate a reggeizing piece:

$$
D_{6}^{I}=D_{6}^{I ; R}+D_{6}^{I ; I}
$$

and rewrite the equation. Our ansatz for the first term, $D_{6}^{I ; R}$, will be constructed in such a way that, to lowest order, it removes the $L$ and the $I+J$ terms in (3.11), (3.14), and (3.15). We put:

$$
D_{6}^{I ; R}=D_{6}^{I ; R(I+J)}+D_{6}^{I ; R(L)}
$$

with the two terms being illustrated in figure $8(\mathrm{a})$ and (b): figure 8 a belongs to the partition (12)(3456), figure 8(b) to the partition (123)(456). Both terms contain the vertex $V_{4 \leftarrow 2}$ which, according to $(2.22)$, consists of three terms. But in the large- $N_{c}$ limit and for our color configuration, only the first term contributes, in which the pairs (ab) and (cd) are color singlet states. Moving further down in figure 8, we recognize two separate BFKL ladders; this separation is a consequence of the large- $N_{c}$ limit. For the first term, figure 8a, we have at the bottom the color and momentum structure described in (3.14)-(3.17). The 'L'-term in figure 8(b), described in (3.11)-(3.13), consists of products of two triplets, and in each triplet we have a sum of three terms.

We emphasize that the reggeizing pieces have been constructed in such a way that, to lowest order, they coincide with the second and third terms on the r.h.s. of figure 7. Taking 

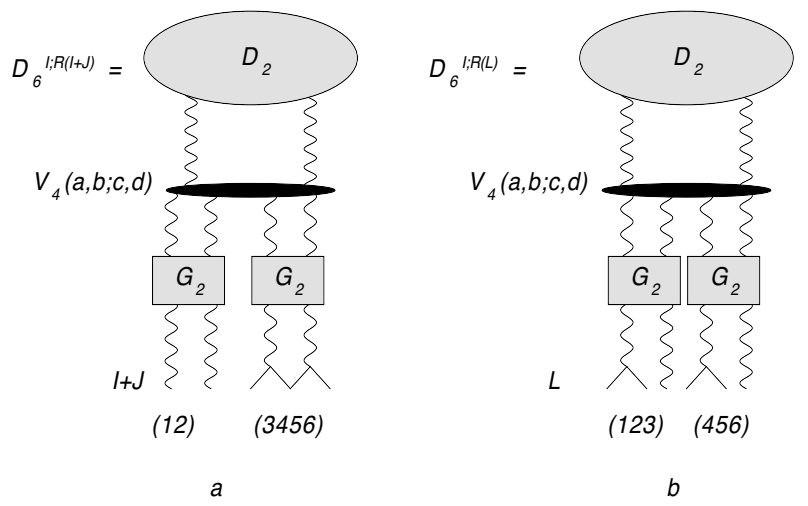

Figure 8. Reggeizing pieces of the second reduction, $D_{6}^{I ; R}$ : (a) $D_{6}^{I ; R(I+J)}$, (b) $D_{6}^{I ; R(L)}$. As in figure 6 , the pairs of straight lines at the lower end of reggeized gluons indicate that they depend upon the sum of momenta and finally decay into two gluons. Further details are described in section 4.4 .

into account the subsequent large- $N_{c}$ evolution, this requirement fixes the reggeizing pieces uniquely. Further below (in section 4.4) we will come back to these reggeizing terms and discuss in more detail their significance.

\subsection{The large- $N_{c}$ limit of eq. (3.9)}

With the ansatz (4.1) and (4.2) we return to (3.9) (or figure 7) and insert it into the terms containing $D_{6}^{I}$ :

$$
\begin{aligned}
S_{6}\left(D_{6}^{I ; R(L)}+D_{6}^{I ; R(I+J)}+D_{6}^{I ; I}\right)= & W_{\text {odd }} D_{2}+T_{L} D_{2}+\left(T_{I}+T_{J}\right) D_{2}+\sum K_{3 \leftarrow 2} D_{5}^{I} \\
& +\sum K_{4 \leftarrow 2} D_{4}^{I}+\sum_{(i j)} V_{i j}\left(D_{6}^{I ; R(L)}+D_{6}^{I ; R(I+J)}+D_{6}^{I ; I}\right) .
\end{aligned}
$$

Our goal is the derivation of an equation for $D_{6}^{I ; I}$. As it was said before, in this paper we consider the configuration where the six gluon state consists of three color singlet pairs, and we consider the large- $N_{c}$ limit of the corresponding six gluon amplitude. To be definite, we demand that the pairs (12), (34), and (56) are in color singlet states. This allows for the three partitions (12)(3456), (1234)(56), and (1256)(34).

We proceed by discussing, on the r.h.s. of (4.3), term by term. We will find that for our color configuration, in the large $N_{c}$ limit the r.h.s. simplifies. For reasons which will become clear soon we begin with the pieces containing $D_{5}^{I}$ and the $2 \rightarrow 3$ kernel. From (3.7) we see that $D_{5}^{I}$ is a sum of 10 terms. Each term contains a $V_{4 \leftarrow 2}$ vertex. Below this vertex we have the evolution of the four reggeon state, which in the large- $N_{c}$ limit splits into the evolution of two independent two-reggeon states (2.24). At the end of this evolution one of the four reggeized gluons splits into two gluons. Subsequently, we attach a vertex with $K_{3 \leftarrow 2}$. This structure implies that the $D_{5}$ term on the r.h.s. of figure 7 can be written as a sum of different groups of contributions: they are illustrated in figure 9. In the first group we have the partition (12)(3456), where the subsystem (12) evolves independently, and the 

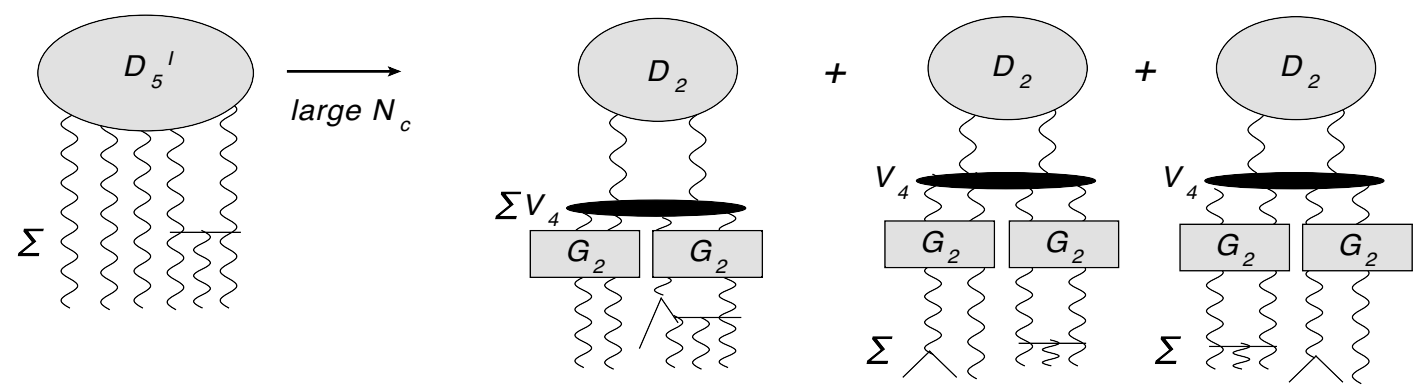

Figure 9. The three terms emerging from the $D_{5}^{I}$ term in figure 7 . The first term belongs to the partition (12)(3456), the second and third ones to the partition (123)(456).

$2 \rightarrow 3$ transition is inside the subsystem (3456). Second, in the partition (123)(456) there exists the part of $D_{5}^{I}$ which contains the splittings (12), (23), and (13), and the $2 \rightarrow 3$ transition sits inside the triplet (456). In the third group, the $2 \rightarrow 3$ transition is inside the triplet (123). Consequently, only the first group contributes to our configuration of three color singlet pairs, whereas the last two terms have triplet structures. As we will discuss below, they have to be combined with the $L$-terms and are not important for the following discussion. Let us discuss the first group in more detail. Beginning with $D_{5}^{I}$ in (3.7), a color singlet of the pair (12) can come only from the last three terms:

$$
\begin{gathered}
D_{5}^{I, a_{1} a_{2} a_{3} a_{4} a_{t}} \rightarrow \frac{g}{2}\left[f^{a_{3} a_{4} c} D_{4}^{I, a_{1} a_{2} c a_{5}}(1,2,34,5)+f^{a_{3} a_{5} c} D_{4}^{I, a_{1} a_{2} c a_{4}}(1,2,35,4)\right. \\
\left.+f^{a_{4} a_{5} c} D_{4}^{I, a_{1} a_{2} a_{3} c}(1,2,3,45)\right] .
\end{gathered}
$$

In the first term we have

$$
D_{4}^{I}(1,2,34,5)=\left(G_{4} V_{4 \leftarrow 2} D_{2}\right)(1,2,34,5) .
$$

As we have said before, the evolution in $G_{4}$ factorizes:

$$
G_{4}(1,2,3,4)=G_{2}(1,2) G_{2}(3,4),
$$

and we arrive at:

$$
G_{2}(1,2) G_{2}(34,5)\left(V_{4} D_{2}\right)(1,2 ; 34,5) \delta_{a_{1} a_{2}} f^{a_{3} a_{4} a_{5}},
$$

where the Green's functions $G_{2}(1,2)$ and $G_{2}(34,5)$ (suppressing the color factors) describe the pomeron evolution in the subsystems $(1,2)$ and $(34,5)$ resp. Together with analogous expressions for the other two terms in (4.4) we find:

$$
\begin{aligned}
& D_{5}^{I, a_{1} a_{2} a_{3} a_{4} a_{t}} \rightarrow \\
&=\frac{g}{2} \delta_{a_{1} a_{2}} f^{a_{3} a_{4} a_{5}} G_{2}(1,2)[ G_{2}(34,5)\left(V_{4} D_{2}\right)(1,2 ; 34,5)-G_{2}(35,4)\left(V_{4} D_{2}\right)(1,2 ; 35,4) \\
&\left.+G_{2}(3,45)\left(V_{4} D_{2}\right)(1,2 ; 3,, 45)\right] .
\end{aligned}
$$

Apart from the extra Green's function $G_{2}(1,2)$ we have the same structure as $D_{3}$ in (2.12). We attach the $2 \rightarrow 3$ vertex:

$$
\begin{aligned}
T_{2} & =\sum\left(K_{3 \leftarrow 2} D_{5}^{I}\right) \\
& =G_{2}(1,2) \sum\left(K_{3 \leftarrow 2} G_{2}\left(3^{\prime}, 4^{\prime}\right)\right)\left(V_{4} D_{2}\right)\left(12,3^{\prime}, 4^{\prime}\right) .
\end{aligned}
$$


As the next term on the rhs (4.3) we consider the triplet states. The first term on the r.h.s. of (4.3) contains the pomeron $\rightarrow 2$ odderon vertex. Its structure is explained in (3.10): the two gluon state splits into two triplet states which are characterized by the symmetric $d^{a_{1} a_{2} a_{3}}$ tensors. As an example, we once more chose the partition (123)(456) at the $W$ vertex in (3.10). Below this vertex, the two triplet states, in the large- $N_{c}$ approximation, evolve separately and do not mix. Each of them forms an QCD odderon state, as described for example, in [19-21].

A similar discussion applies to the second term on the r.h.s. of (4.3), the $L$-term (cf. (3.11)-(3.13)): below the vertex $V_{4 \leftarrow 2}$ two triplet states start, this time accompanied with $f$-tensors. As written in (3.12)-(3.13), in each of the two triplets the momentum structure is the same as for $D_{30}$ in (2.9). These terms represent the inhomogeneous terms, the last two terms in figure 9 the kernels for the evolution equation of the reggeizing terms $D_{6}^{I ; R(L)}$. They exactly equal the $D_{6}^{I ; R(L)}$ term on the l.h.s. of the integral equation (4.3):

$$
S_{6} D_{6}^{I ; R(L)}=T_{L} D_{2}+\left.\sum K_{3 \rightarrow 2} D_{5}^{I}\right|_{\text {last two terms in figure } 8}+\sum_{(i j)} V_{i j} D_{6}^{I ; R(L)} .
$$

We note that these triplet terms, at large $N_{c}$, do not contribute to the color structure we are considering in this paper. However we note that the terms with the f-structure will be crucial when we turn to the next step, the case of 8 gluons. Here we expect new fan diagrams: in addition to the fan diagram in figure 2 we should find a higher order fan diagram where the lower BFKL ladder on the l.h.s. will split into two ladders. This is where the ' $\mathrm{L}$ ' terms will be needed for building the two lower triple pomeron vertices.

Returning to the r.h.s. of (4.3) we, from now on, disregard the odderon term on both sides of the equation: as an aditive contribution to $D_{6}^{I}$ it does not mix with the other pieces and satisfies its own equation. Similarly, we remove $D_{6}^{I ; R(L)}$ on the both sides of the equation, together with the $T_{L} D_{2}$ terms and parts of the $D_{5}$ contributions on the r.h.s. We are thus left with the combination $D_{6}^{I ; R(I+J)}+D_{6}^{I ; I}$, where $D_{6}^{I ; I}$ now is without the two-odderon piece (for simplicity, we continue to use the same notation).

Let us then take a closer look at the remaining pieces. We begin with the terms $T_{I}$ and $T_{J},(3.14)-(3.17)$ and focus on the partition (12)(3456)... For this partition, the couplings at the vertex $V_{4 \leftarrow 2}$ have the color structure $\delta_{a_{1} a_{2}} d^{a_{3} a_{4} a_{5} a_{6}}$. and the momentum structure described in (3.16), (3.17). Considering the four reggeons ' 3 ', ' 4 ', ' 5 ', and ' 6 ', the structure of the 7 terms in the sum 'I' and ' $\mathrm{J}$ ' is exactly the same as for $D_{40}$ in (2.13). Reggeons ' 1 ' and ' 2 ' play the role of spectators:

$$
T_{1}=\left(\left(T_{I}+T_{J}\right) D_{2}\right)(1,2 ; 3,4,5,6) .
$$

Turning to the terms containing $\left.D_{4}^{(} I\right)$ (the next-to-last term in figure 7 ), we note that the evolution of the four gluon state in $D_{4}^{I}$ is the sum of three states, (12)(34), (13)(24), and (14)(23), and in each of them the evolution is described by two separate BFKL Green's functions. Only the first term belongs to the color singlet (12), and consequently the $2 \rightarrow 4$ kernel has to be inside the quartet (3456). In rapidity space this term can be written in the following form:

$$
T_{3}=\int d y^{\prime} G_{2}^{(12)}\left(y_{1}-y^{\prime}\right) K_{4 \leftarrow 2} G_{2}^{\left(3^{\prime} 4^{\prime}\right)}\left(y_{2}-y^{\prime}\right)\left(V_{4} D_{2}\left(y^{\prime}\right)\right)\left(1,2 ; 3^{\prime} 4^{\prime}\right) .
$$




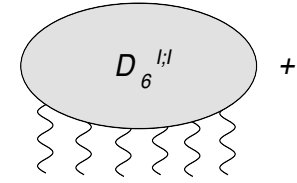

(12) (3456)

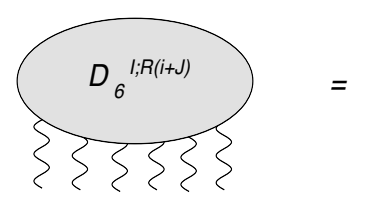

(12) (3456)

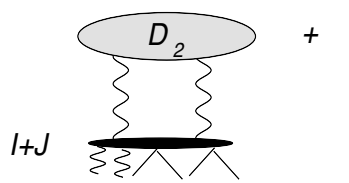

(12) (3456)

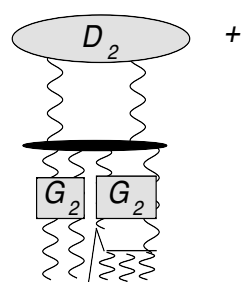

(12) (3456)

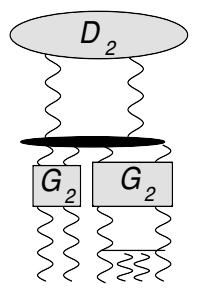

(12) (3456)

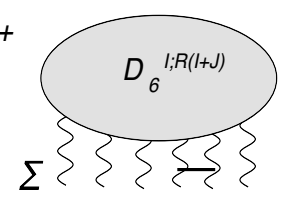

(12) (3456)

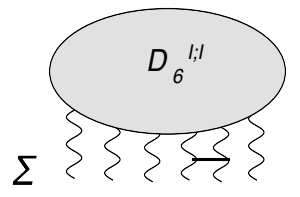

(12) (3456)

$$
T_{1} T_{2} \quad T_{3}
$$

Figure 10. The integral equation for the combination $D_{6}^{I ; R(I+J)}+D_{6}^{I ; I}$, restricted to the partition (12)(3456). Here, in the last two terms, the sum over pairwise interaction includes only the pair (12) and pairwise interactions inside the quartet (3456).

This finishes our discussion of the inhomogeneous terms on the r.h.s. of (4.3), For the partition $(12)(3456)$ we therefore can write the following integral equation for the combination $D_{6}^{I ; R(I+J)}+D_{6}^{I ; I}$ :

$$
S_{6}\left(D_{6}^{I ; R(I+J)}+D_{6}^{I ; I}\right)=T_{1}+T_{2}+T_{3}+\sum_{(i j)} V_{i j}\left(D_{6}^{I ; R(I+J)}+D_{6}^{I ; I}\right) .
$$

We illustrate this equation in figure 10.

\subsection{Two triple pomeron vertices}

Let us now analyze the integral equation illustrated in figure 10. Most strikingly, we notice that the subsystem (12) has been separated from the subsystem (3456): the term $D_{6}^{I ; R(I+J)}$ on the 1.h.s. and on the r.h.s. (illustrated in figure 8(a)), as well as the first three terms in the second line of figure 10 all contain $V_{4}$, and below we have a separate cylinder for the (12) subsystem. To see this in more detail, we first note that the reggeizing terms $D_{6}^{I ; R(I+J)}$ (figure $8(\mathrm{a})$ ) in rapidity space can be written as

$$
\int d y^{\prime} G_{2}^{(12)}\left(y_{1}-y^{\prime}\right) \sum G_{2}^{(34,56)}\left(y_{2}-y^{\prime}\right)\left(V_{4} D_{2}\left(y^{\prime}\right)\right)\left(1,2 ; 3^{\prime} 4^{\prime}\right)
$$

Here the sum in front of $G_{2}^{(34,56)}\left(y_{2}-y^{\prime}\right)$ stands for summing, at the lower end of the BFKL evolution, over momentum and color structures, in accordance with (2.13). Interpreting the rapidity evolution in (4.14, we compare with figure 8a: starting from above, we evolve up to rapidity $y^{\prime}$ where the two-gluon state merges into the four gluon state. Below, the 
pair (12) evolves up to $y_{1}$, whereas the other two-gluon state evolves up to $y_{2}$ where it splits into the four gluons (3456).

Next, for the inhomogeneous terms $T_{1}+T_{2}+T_{3}$ we note that - formally - we could solve the integral equation (4.13) by introducing the Green's function $G_{6}$ : in rapidity space we put

$$
S_{6}=\partial_{y}+\sum_{i=1}^{6} \omega(i)
$$

and (4.13) can be solved by

$$
\left(S_{6}-\sum_{(i j)} V_{i j}\right)\left(D_{6}^{I ; R(I+J)}+D_{6}^{I ; I}\right)=T_{1}+T_{2}+T_{3}
$$

or

$$
\left(D_{6}^{I ; R(I+J)}+D_{6}^{I ; I}\right)=G_{6}\left(T_{1}+T_{2}+T_{3}\right)
$$

with

$$
\left(S_{6}-\sum_{(i j)} V_{i j}\right) G_{6}(y)=\delta(y) .
$$

In the large- $N_{c}$ limit there is no interaction between the systems (12) and (3456), such that in the Green's function we can simplify:

$$
S_{6}-\sum_{(i j)} V_{i j} \rightarrow S_{6}-V_{12}-\sum_{3 \leq i, j} V_{(i j)} .
$$

Consequently, the Green's function $G_{6}$ splits into a product:

$$
G_{6}(1,2,3,4,5) \rightarrow G_{2}(1,2) G_{4}(3,4,5,6),
$$

where each of the factors satisfies its own equation:

$$
\left(S_{2}-V_{12}\right) G_{2}(y ; 1,2)=\delta(y),\left(S_{4}-\sum_{3 \leq i, j} V_{(i j)}\right) G_{4}(y ; 3456)=\delta(y) .
$$

We now use this decomposition to evaluate the three terms on the r.h.s. of (4.17). For $T_{1}$ we find:

$$
\begin{aligned}
\int d y^{\prime} G_{2}\left(y_{1}-y^{\prime} ; 12\right) G_{4}\left(y_{2}-y^{\prime}, 3456\right) T_{1}\left(y^{\prime}\right) \\
=\int d y^{\prime} G_{2}\left(y_{1}-y^{\prime} ; 12\right) G_{4}\left(y_{2}-y^{\prime}, 3456\right)\left(T_{I}+T_{J}\right) D_{2}\left(y^{\prime}\right) .
\end{aligned}
$$

Comparison with figure 10 shows that the transition from 2 to 4 gluons occurs at rapidity $y^{\prime}$.

For the other two terms, $T_{2}$ and $T_{3}$, the situation is a bit more complicated since they contain already evolution inside the subsystem (3456). Using the identity

$$
\int d y^{\prime} G_{2}\left(y_{1}-y^{\prime}\right) G_{2}\left(y^{\prime}-y_{2}\right)=G_{2}\left(y_{1}-y_{2}\right)
$$


we write for the $T_{2}$ term:

$$
\begin{array}{r}
\int d y^{\prime} G_{2}\left(y_{1}-y^{\prime} ; 12\right)\left(\int d y^{\prime \prime} G_{4}\left(y_{2}-y^{\prime \prime} ; 3456\right) \sum\left(K_{3 \leftarrow 2} G_{2}\left(y^{\prime \prime}-y^{\prime} ;\left(3+3^{\prime}\right) 4^{\prime}\right)\right)\right) . \\
\cdot\left(V_{4} D_{2}\left(y^{\prime}\right)\right)\left(12,3^{\prime} 4^{\prime}\right) .
\end{array}
$$

Similarly, for the $T_{3}$ term

$$
\begin{aligned}
& \int d y^{\prime} G_{2}\left(y_{1}-y^{\prime} ; 12\right)\left(\int d y^{\prime \prime} G_{4}\left(y_{2}-y^{\prime \prime} ; 3456\right) K_{4 \leftarrow 2} G_{2}\left(y^{\prime \prime}-y^{\prime} ; 3^{\prime} 4^{\prime}\right)\right) . \\
& \cdot\left(V_{4} D_{2}\left(y^{\prime}\right)\right)\left(12,3^{\prime} 4^{\prime}\right) .
\end{aligned}
$$

Again, figure 10 provides an illustration of these two equations.

All three terms have the common Green's function in the subsystem (12). In order to fully separate the evolution of the pair (12), we choose $y_{1}<y_{2}$, fix the rapidity $y^{\prime}=y_{1}$, and use the property

$$
G_{2}(0)=1,
$$

i.e. the pair (12) does not evolve in rapidity. This then leads to the following expression for the r.h.s. of (4.17):

$$
\begin{aligned}
& \left.G_{6}\left(T_{1}+T_{2}+T_{3}\right)\right|_{y^{\prime}=y_{1}}= \\
& \quad G_{4}\left(y_{2}-y_{1} ; 3456\right)\left(T_{I}+T_{J}\right) D_{2}\left(y_{1}\right) \\
& \quad+\left(\int d y^{\prime \prime} G_{4}\left(y_{2}-y^{\prime \prime} ; 3456\right) \sum\left(K_{3 \leftarrow 2} G_{2}\left(y^{\prime \prime}-y_{1} ;,\left(3+3^{\prime}\right) 4^{\prime}\right)\right)\right)\left(V_{4} D_{2}\left(y_{1}\right)\right)\left(12,3^{\prime} 4^{\prime}\right) \\
& \quad+\left(\int d y^{\prime \prime} G_{4}\left(y_{2}-y^{\prime \prime} ; 3456\right) K_{4 \leftarrow 2} G_{2}\left(y^{\prime \prime}-y_{1}\right)\right)\left(V_{4} D_{2}\left(y_{1}\right)\right)\left(12,3^{\prime} 4^{\prime}\right)
\end{aligned}
$$

This equation has to be compared with the solution of $D_{4}$ in eq. (2.17). On the r.h.s. of (4.27 we keep the momenta of the pair (12) fixed and concentrate on the subsystem (3456) below the upper triple pomeron vertex, i.e. $V_{4} D_{2}\left(y_{1}\right)$. Then we see, term by term, that the r.h.s. of (4.27) is the same as of (2.17), provided we substitute $D_{20} \rightarrow\left(T_{I}+T_{J}\right) D_{2}$. The second and third lines on the r.h.s. of (4.27) correspond to the second and third terms on the r.h.s. of (2.17), once we take into account that $\sum K_{3 \leftarrow 2} D_{3}$ in (2.17) really means the same as $\sum K_{3 \leftarrow 2} G_{2}$ in (4.27).

We then make use of the discussion given after (2.17). For the subsystem (3456) below $V_{4} D_{2}\left(y_{1}\right)$ we have the same structure as for $D_{4}$, i.e. we have the sum of reggeizing piece and an irreducible one. The former one has the form:

$$
\sum G_{2}^{(34,56)}\left(y_{2}-y_{1}\right)\left(V_{4} D_{2}\left(y_{1}\right)\right)\left(1,2 ; 3^{\prime} 4^{\prime}\right)
$$

where the symbol $\Sigma$ reminds that the Green's function $G_{2}^{(34,56)}\left(y_{2}-y_{1}\right)$ at its lower end has the same momentum structure as $D_{4}^{R}$. The second term, the analogue of $D_{4}^{I}$, has the 


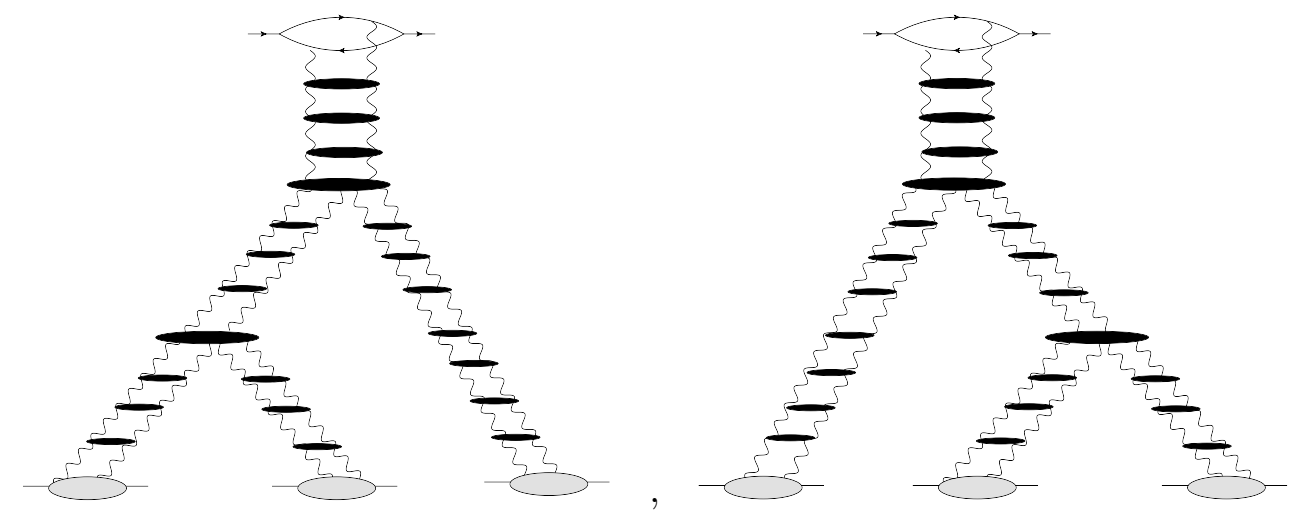

Figure 11. Two more fan diagrams.

form:

$$
\int d y^{\prime \prime} G_{2}\left(y_{2}-y^{\prime \prime} ; 34\right) G_{2}\left(y_{2}-y^{\prime \prime} ; 56\right) V_{4} G_{2}\left(y^{\prime \prime}-y_{1} ; 3^{\prime \prime} 4^{\prime \prime}\right)\left(V_{4} D_{2}\left(y_{1}\right)\right)\left(12,3^{\prime} 4^{\prime}\right) .
$$

Finally we re-introduce the integration of $y^{\prime}$ and the Green's function for the pair (12). The first part then takes the form

$$
\int d y^{\prime} G_{2}^{(12)}\left(y_{1}-y^{\prime}\right) \sum G_{2}^{(34,56)}\left(y_{2}-y^{\prime}\right)\left(V_{4} D_{2}\left(y^{\prime}\right)\right)\left(1,2 ; 3^{\prime} 4^{\prime}\right)=D_{6}^{I ; R(I+J)}\left(y_{1}, y_{2}\right)
$$

and thus coincides with the reggizing piece $D_{6}^{I ; R(I+J)}$ in (4.14). The second term becomes:

$$
\begin{aligned}
D_{6}^{I ; I}\left(y_{1}, y_{2}, y_{3}\right)= & \int d y^{\prime} G_{2}\left(y_{1}-y^{\prime} ; 12\right) \int d y^{\prime \prime} G_{2}\left(y_{2}-y^{\prime \prime} ; 34\right) G_{2}\left(y_{3}-y^{\prime \prime} ; 56\right) \\
& \cdot V_{4} G_{2}\left(y^{\prime \prime}-y^{\prime} ; 3^{\prime \prime} 4^{\prime \prime}\right)\left(V_{4} D_{2}\left(y^{\prime}\right)\right)\left(12,3^{\prime} 4^{\prime}\right)
\end{aligned}
$$

and has the fan structure illustrated in figure 2. Therefore we conclude that the r.h.s. of (4.27) which had been obtained from evaluating the r.h.s. of (4.17) is, in fact, the sum

$$
D_{6}^{I}=D_{6}^{I ; R(I+J)}+D_{6}^{I ; I}
$$

with $D_{6}^{I ; R(I+J)}$ in (4.14) and $D_{6}^{I ; I}$ in (4.31). The fan structure in eq. (4.31) (figure 2) represents the main result of this paper.

For completeness a few words need still to be said about the remaining two partitions, (1234)(56) and (1256)(34). By going through the same sequence of arguments as described for the partition (12)(3456) one arrives at the fan diagrams shown in figure 11. It is only after adding these two additional fan diagram that the Bose symmetry of pomeron diagrams is restored.

\subsection{Reggeizing pieces in the four and six giuon amplitudes $D_{4}$ and $D_{6}$}

Finally we want to comment on the reggeizing terms, $D_{4}^{R}, D_{6}^{R}$, and $D_{6}^{I ; R(I+J)}$ and $D_{6}^{I ; R(L)}$. In our derivation, they appear as additive corrections to the 'simple' fan diagrams, and they require an interpretation. 
Let us first return to $D_{4}^{R}$, which we have discussed already at the end of section 2.2 . First a word about the order of this term. Compared to $D_{2}, D_{4}^{R}$ is of a higher order in the coupling constant: it contains an extra $\alpha_{s}$. Projecting onto the target color configuration where the two pairs (12) and (34) form singlets, it has the order $\alpha_{s}^{2} N_{c}^{3}\left(1+\sum_{n=1} \alpha_{s} N_{c} y\right)$ where the sum is provided by evolution. This can be compared to the contribution from the triple pomeron, which has the order $\alpha^{3} N_{c}^{4} y\left(1+\sum_{n=1} \alpha_{s} N_{c} y\right)$ where again the sum is given by evolution. As one observes these two contributions are of the same order provided $\alpha_{s} N_{c} y \sim 1$ which is assumed in the leading logarithmic approximation. Separating the contribution from the impact factor of the order $\alpha_{s} N_{c}$ one observes that the triple pomeron without evolution has the same order as the reggeized term with a single interaction inside the BFKL chain.

Next we recapitulate our interpretation of $D_{4}^{R}$. Starting from its simplest form, the coupling of a BFKL pomeron to the nucleus is given by the coupling to a single nucleon inside the nucleus, and this coupling is described by the gluon density of the nucleon. Following this picture, $D_{4}^{R}$ in figure 6(b) describes the coupling of the pomeron to a pair of nucleons, and in [18] it has been shown that this can be intepreted as the second term in an eikonal expansion of the initial conditions of the nonlinear evolution equations. In the original derivation of the BK equation [9] the low-energy scattering of the $q \bar{q}$ loop on the nucleus in the nucleus rest frame was taken in the natural eikonal form, following the lowest order (classical) picture [22]. The interpretation of $D_{4}^{R}$ given in [18] clearly corresponds to this picture.

However the validity of this picture also for the projectile proton is not so obvious, especially when one wants to apply the saturation momentum approach to the proton itself. So in practical calculations for hA and AB scattering within the JIMWLK approach certain more phenomenological initial conditions were taken, guided by the experimental data, e.g. in [23-25]. They do not eikonalize the scattering on the nucleus in terms of the scattering on the proton at low energies, and so they imply a non-standard spatial picture of the large heavy nucleus as composed of individual nucleons.

For completeness we have to include into our discussion also $D_{3}$ (figure 4) and $D_{5}^{R}$. In $D_{3}$, one of the lower reggeons decays into two elementary gluons: we interpret this as a higher order correction to the lowest order coupling of the BFKL pomeron to a nucleon. Similarly, $D_{5}^{R}$ has the same structure as $D_{4}^{I}$, where one of the two lower pomerons has the properties of $D_{3}$.

In $D_{6}$ we again have found reggeizing terms: $D_{6}^{R}$ in (3.8), and $D_{6}^{I ; R}$ in (4.1) which, in fact, consisted of the two classes of contributions $(4.2), D_{6}^{I ; R(I+J)}$ and $D_{6}^{I ; R(L)}$. The first term, $D_{6}^{R}$ with the momentum structure given in (3.2), belongs to a single BFKL pomeron which decays into six elementary gluons and thus contains couplings to (up to) three nucleons. As an example we illustrate in figure 12a the term, $D_{2}(135,246)$. This term has to been seen in the context of $D_{4}^{R}$ : the pomeron now couples to a triplet of nucleons: this supports our previous finding that reggeizing terms provide a nontrivial structure for the couplings of pomerons to the nucleus.

The detailed structure of $D_{6}^{I ; R(I+J)}$ is contained in (3.16) and (3.17), the structure of $D_{6}^{I ; R(L)}$ in (3.13). Again, we illustrate examples. For the $I+J$ structure we choose, 


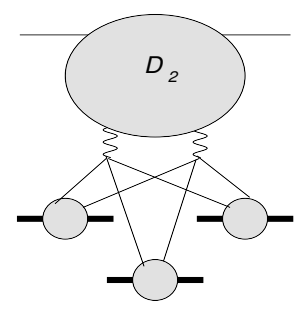

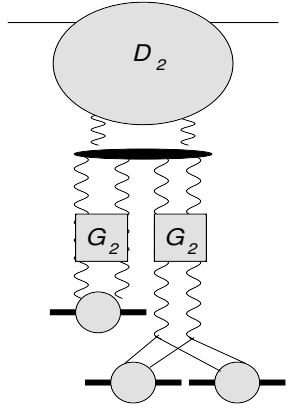

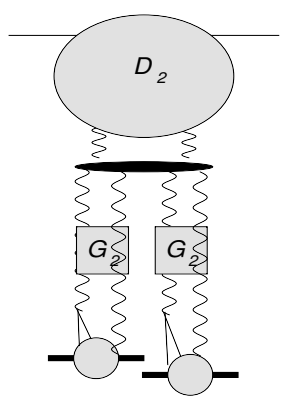

Figure 12. Reggeized terms in $D_{6}$ : (a) a term in $D_{6}^{R}$ in $(3.8) ;\left(\right.$ b) a term in $D_{6}^{I ; R(I+J)}$;(c) a term in $D_{6}^{I ; R(L)}$.

in figure 12(b), the term with the momentum structure $(1,2 ; 35,46)$. As one can see from (3.16) and (3.17), the right BFKL pomeron at the lower end has exactly the same structure as the single pomeron in $D_{4}^{R}$ and thus couples to the nucleus in the same manner as the single pomeron in $D_{4}^{R}$. In figure $12(\mathrm{~b})$ we show the analogue of figure $6(\mathrm{~b})$, the coupling to a pair of nucleons. As to the L-structure, a look at (3.13) shows that now each of the pomerons at the lower end has the structure of $D_{3}$ : an example is shown in figure 12c. In summary, both sets of reggeizing contributions, $D_{6}^{I ; R(I+J)}$ and $D_{6}^{I ; R(L)}$, have the same structure as $D_{4}^{I}$, but the two lower pomerons are corrected: either both pomerons decay into three gluons and have the structure of $D_{3}$, or one of them decays into four gluons and repeats $D_{4}^{R}$ whereas the other one remains uncorrected.

Let us summarize our main findings for $D_{2}, D_{4}$ and $D_{6}$ in figure 13 (see also figure 6(b) and 12(a)). In addition to the 'simple' fan structure (on the lhs) where each pomeron at the lower end couples to a single nucleon inside the nucleus, we found reggeizing pieces (on the rhs) which are interpreted as coupling to two or more nucleons and can be absorbed into suitable intitial condtions.

These results suggest the following generalization to amplitudes $D_{2 n}$ with $2 n$ reggeons at the lower end: in addition to the fan diagrams with $n$ pomerons at the lower end we should find a hierarchy of reggeizing pieces: single pomerons which decay into $2 n$ elementary gluons and thus can couple to up to $n$ nucleons, the first fan diagram with two pomerons which can decay into $(2,2(n-2)),(3,(2 n-3))$ gluons etc.

We consider the existence of our reggeizing pieces, together with their interpretation, as quite an important result of our analysis. Namely, such contributions related to the reggeization of the gluons do not show up in derivations based upon the dipole picture. However, a closer look at the derivation of the BK-equation [9] shows that the situation is more subtle. Namely, in addition to the nonlinear evolution which is derived within in the dipole picture, a specific form of the coupling to nuclei is also included; in coordinate space this coupling has an eikonal structure, and when expanded it describe the coupling to one, two, three nucleons, similar to the first line of figure 13. From what we can say at the moment (but as we have emphasized before, the detailed analysis of our couplings still 

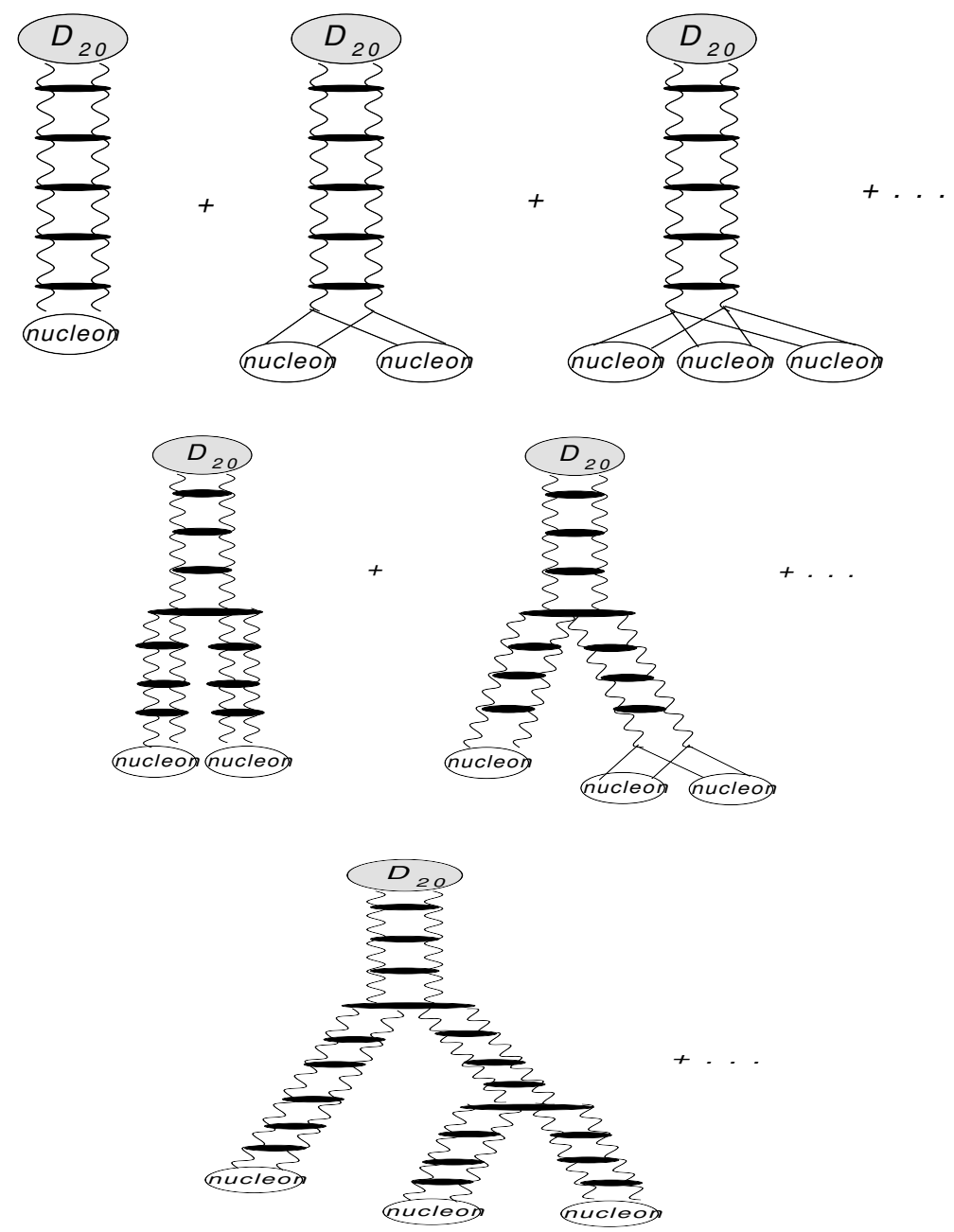

Figure 13. Illustrating our main results: in the upper line the single pomeron can couple to one, two, three nulceons; in the second line we show the first fan diagram with couplings to one, two nucleons; in the third line we have the second order fan diagrams with couplings to single nucleons.

needs some further investigation), both approaches seem to arrrive at the consistent result:

- our derivation (performed in perturbation theory in momentum space) leads to contributions which arise from the reggization of the gluon but can be reformulated as specific corrections in the couplings of the pomerons to the nuclei;

- the derivation based upon the color dipole picture does not see such 'reggeized' contributions, but by including the nontrivial form of couplings of pomerons to the nuclei it leads to the same result.

Clearly, a more detailed analysis of this equivalence is highly desirable and we hope to come back to this in a future publication. 


\section{Discussion}

In our study we have used the framework of QCD field theory of reggeized gluons for discussing, in the large- $N_{c}$ limit, diagrams with up to six reggeized gluons, using the results of [1]. Our main interest was the formation of two consecutive triple pomeron vertices. We have found that in diagrams with higher splitting kernels $K_{n \leftarrow 2}$ with $n=5$ and 6 all those contributions are present which are needed to form the triple pomeron vertex at each splitting. This result agrees with the conclusions derived in the framework of the dipole model, the Balitski-Kovchegov equation.

In the course of our analysis a few other terms appeared, which require some attention. First, our analysis contains the pomeron $\rightarrow$ two odderon vertex (see the discussion in section 3 , after (3.10)). This vertex was found as early as in 1999, when only the Janik-Wosiek solution (JW) for the odderon [20,21] was known, describing a C-odd bound state of three reggeized gluons. Soon afterwards a new solution with intercept one was found in [19] (the BLV odderon). This solution has a simple quasi-two-gluon structure corresponding to the fusion of two of the three reggeized gluons into a single object (the zero range "digluon"). Since the structure of this odderon is practically identical to the pomeron, it became possible to radically simplify vertices for both pomeron $\rightarrow$ two odderon and odderon $\rightarrow$ odderon+pomeron transitions, which actually reduce to the triple pomeron form. This allowed to easily derive the coupled system of evolution of the pomeron and odderon including both transitions [15, 16]. This system was analyzed in [26] where the angular dependence introduced by the pomeron $\rightarrow$ two odderon transition (POO) was drastically simplified. Recently this very system was investigated in detail and at finite $N_{c}$ [27]. Calculating the pomeron-odderon (PO) evolution the authors neglected the POO term and limited themselves to the lowest azimuthal harmonic. A posteriori, by calculating with the previously obtained solution the POO term the authors then verified that the latter was small. In [27] the full JIMWLK equation was solved numerically by Langevin simulations on the lattice, assuming a certain choice of azimuthally unsymmetric initial conditions which generate odderon contibutions. It was found that the results were in very good agreement with those obtained form the previously studied coupled PO system of equations. It might very well be (although not shown explicitly) that the full JIMWLK system contains the POO transitions not only for the BLV odderon but also the JW odderon solutions. Compared to these important numerical studies, we find it important to stress that the pomeron $\rightarrow$ two odderon vertex found in [1] and in our present analysis, describing the full transition from 2 to 6 reggeized gluons, is not restricted to the BLV form of the odderon but is valid also for a more general odderon consisting of three gluons, such as the JW odderon. It would be interesting to study the coupled evolution of pomerons and odderon with the full angular dependence and the JW odderon as well.

Second, our investigation has led us to separate, from the pomeron fan diagrams, an extra class of contributions which result from the reggeization of the gluon ('reggeized terms'). A detailed discussion has been given in section 4.4 and the main results are summarized in figure 13. The first example, for the case of four reggeized gluons, was $D_{4}^{R}$ (figure 6(b)). For the six-gluon case we first found $D_{6}^{R}$ (see (3.8). As illustrated in the 
first line of figure 13 (see also figure 6(b) and figure $12(\mathrm{a})$ ), both $D_{4}^{R}$ and $D_{6}^{R}$, contain the two gluon state of the pomeron, $D_{2}$, which at the lower end splits into four or six gluons, resp. To be complete we have to include $D_{3}$ (defined in (2.12)) and $D_{5}^{R}$ (given in (3.5)), where $D_{2}$ splits into three or 5 gluons, resp. Within a nucleus, all these contributions can be interpreted as couplings of a single pomeron to pairs, triplets,... of nucleons inside the nucleus. Somewhat later, figure 8 , we encountered new reggeizing pieces, $D_{6}^{I ; R}$. As an example, $D_{6}^{I ; R(I+J)}$ in figure $8($ a) has the form of the lowest order fan diagram (second line of figure 13), with one of the two lower pomerons splitting into 4 gluons. It thus seems as if the reggeizing pieces, being fully compatible with the fan structure, start to 'dress' the lower order fan diagrams, by introducing nontrivial structures into the coupling of pomerons to the nucleus and thus leading to a result compatible with [9] .

Finally, our investigation of the appearance of more than one triple pomeron vertex within the framework of QCD reggeon field theory based upon reggeized gluons also might serve other purposes. First, it would be interesting to analyze the six gluon system also for finite $N_{c}$. Most likely, a $2 \rightarrow 4$ reggeon vertex for non-singlet color quantum numbers should exist; there is also the possibility of a $4 \rightarrow 6$ reggeon vertex. Our large- $N_{c}$ study might provide help in such an analysis. More general, experience shows that reggeon field theory interaction vertices - either interactions between reggeized gluons or interactions between bound state fields such as the pomeron or the odderon - cannot directly be read off from high energy Feynman diagrams. In the derivation based upon energy discontinuities several steps of 'reduction' have to be performed, as we have demonstrated in this paper. A very attractive way is the use of Lipatov's effective action where the reggeized gluon appears as a fundamental degree of freedom. But also in this framework interactions between reggeized gluons arise only after a careful analysis of several contributions. Examples are the transition vertex $1 \rightarrow 3$ reggeized gluons which has been derived in [28]. It has also been shown that, in accordance with signature conservation, the vertex $1 \rightarrow 2$ reggeized gluons disappears.

An exciting open problem is the appearance of two triple pomeron vertices in the scattering of two nuclei on two nuclei. We hope to address this problem in a future investigation.

\section{Acknowledgments}

One of us (MB) thanks Hamburg University and the II.Institute of Theoretical Physics for the hospitality. One of us (JB) gratefully acknowledges stimulating and helpful discussions with C.Ewerz.

Open Access. This article is distributed under the terms of the Creative Commons Attribution License (CC-BY 4.0), which permits any use, distribution and reproduction in any medium, provided the original author(s) and source are credited. 


\section{References}

[1] J. Bartels and C. Ewerz, Unitarity corrections in high-energy QCD, JHEP 09 (1999) 026 [hep-ph/9908454] [INSPIRE].

[2] J. Bartels, Unitarity corrections to the Lipatov Pomeron and the four gluon operator in deep inelastic scattering in QCD, Z. Phys. C 60 (1993) 471 [INSPIRE].

[3] J. Bartels and M. Wusthoff, The triple Regge limit of diffractive dissociation in deep inelastic scattering, Z. Phys. C 66 (1995) 157 [InSPIRE].

[4] A.H. Mueller, Soft gluons in the infinite momentum wave function and the BFKL Pomeron, Nucl. Phys. B 415 (1994) 373 [inSPIRE].

[5] A.H. Mueller and B. Patel, Single and double BFKL Pomeron exchange and a dipole picture of high-energy hard processes, Nucl. Phys. B 425 (1994) 471 [hep-ph/9403256] [INSPIRE].

[6] M.A. Braun and G.P. Vacca, Triple Pomeron vertex in the limit $N_{c} \rightarrow \infty$, Eur. Phys. J. C 6 (1999) 147 [hep-ph/9711486] [INSPIRE].

[7] J. Bartels, L.N. Lipatov and G.P. Vacca, Interactions of reggeized gluons in the Mobius representation, Nucl. Phys. B 706 (2005) 391 [hep-ph/0404110] [INSPIRE].

[8] I. Balitsky, Operator expansion for high-energy scattering, Nucl. Phys. B 463 (1996) 99 [hep-ph/9509348] [INSPIRE].

[9] Y.V. Kovchegov, Small $x$ F(2) structure function of a nucleus including multiple Pomeron exchanges, Phys. Rev. D 60 (1999) 034008 [hep-ph/9901281] [INSPIRE].

[10] J. Bartels, M.G. Ryskin and G.P. Vacca, On the triple Pomeron vertex in perturbative QCD, Eur. Phys. J. C 27 (2003) 101 [hep-ph/0207173] [INSPIRE].

[11] R.B. Peschanski, Dual Shapiro-Virasoro amplitudes in the QCD dipole picture, Phys. Lett. B 409 (1997) 491 [hep-ph/9704342] [INSPIRE].

[12] M.A. Braun, Pomeron loops in the perturbative QCD with large $N_{c}$, Eur. Phys. J. C 63 (2009) 287 [arXiv:0901.3660] [INSPIRE].

[13] M.A. Braun and A.N. Tarasov, Scattering amplitude and Pomeron loops in perturbative QCD at large $N_{c}$, Phys. Lett. B 726 (2013) 300 [arXiv:1304.3014] [INSPIRE].

[14] M. Braun, Structure function of the nucleus in the perturbative QCD with $N_{c} \rightarrow \infty$ (BFKL Pomeron fan diagrams), Eur. Phys. J. C 16 (2000) 337 [hep-ph/0001268] [INSPIRE].

[15] Y.V. Kovchegov, L. Szymanowski and S. Wallon, Perturbative odderon in the dipole model, Phys. Lett. B 586 (2004) 267 [hep-ph/0309281] [INSPIRE].

[16] Y. Hatta, E. Iancu, K. Itakura and L. McLerran, Odderon in the color glass condensate, Nucl. Phys. A 760 (2005) 172 [hep-ph/0501171] [INSPIRE].

[17] M. Braun, The System of four Reggeized gluons and the three Pomeron vertex in the high color limit, Eur. Phys. J. C 6 (1999) 321 [hep-ph/9706373] [INSPIRE].

[18] M.A. Braun, On the initial condition for evolution of the perturbative QCD Pomeron in the nucleus, Theor. Math. Phys. 148 (2006) 923 [hep-ph/0503041] [INSPIRE].

[19] J. Bartels, L.N. Lipatov and G.P. Vacca, A new odderon solution in perturbative $Q C D$, Phys. Lett. B 477 (2000) 178 [hep-ph/9912423] [INSPIRE].

[20] J. Wosiek and R.A. Janik, Solution of the Odderon problem for arbitrary conformal weights, Phys. Rev. Lett. 79 (1997) 2935. 
[21] R.A. Janik and J. Wosiek, A solution of the Odderon problem, Phys. Rev. Lett. 82 (1999) 1092.

[22] Y.V. Kovchegov and A.H. Mueller, Gluon production in current nucleus and nucleon-nucleus collisions in a quasiclassical approximation, Nucl. Phys. B 529 (1998) 451 [hep-ph/9802440] [INSPIRE].

[23] K. Dusling, F. Gelis, T. Lappi and R. Venugopalan, Long range two-particle rapidity correlations in $A+A$ collisions from high energy QCD evolution, Nucl. Phys. A 836 (2010) 159 [arXiv:0911.2720] [INSPIRE].

[24] A. Dumitru, J. Jalilian-Marian and E. Petreska, Two-gluon correlations and initial conditions for small-x evolution, Phys. Rev. D 84 (2011) 014018 [arXiv:1105.4155] [INSPIRE].

[25] A. Dumitru and E. Petreska, Initial conditions for dipole evolution beyond the McLerran-Venugopalan model, Nucl. Phys. A 879 (2012) 59 [arXiv:1112.4760] [inSPIRE].

[26] L. Motyka, Nonlinear evolution of Pomeron and odderon in momentum space, Phys. Lett. B 637 (2006) 185 [hep-ph/0509270] [INSPIRE].

[27] T. Lappi, A. Ramnath, K. Rummukainen and H. Weigert, JIMWLK evolution of the odderon, Phys. Rev. D 94 (2016) 054014 [arXiv: 1606.00551] [InSPIRE].

[28] M. Hentschinski, The high energy behavior of QCD: The effective action and the triple-Pomeron-vertex, Ph.D. thesis, Hamburg U., 2009. arXiv: 0908.2576 [INSPIRE]. 